\title{
An extension of valid syllogisms to indefinite terms and a reduction of all the conclusive syllogisms to only Barbara, Darapti and Darii
}

To the memory of my sister Cristina Popa, 1948-2018.

DAN CONSTANTIN RADULESCU ${ }^{[0000-0002-0648-8794]}$

Retired, Boynton Beach FL 33435, USA

dancradulesculyahoo.com, schugu.com

\begin{abstract}
One clarifies ideas from two other papers. Denote by $\mathrm{S}^{*}, \mathrm{P}^{*}, \mathrm{M}^{*}$, where $\mathrm{S}^{*} \in\left\{\mathrm{S}, \mathrm{S}^{\prime}\right\}, \mathrm{P}^{*} \in\left\{\mathrm{P}, \mathrm{P}^{\prime}\right\}, \mathrm{M}^{*} \in\left\{\mathrm{M}, \mathrm{M}^{\prime}\right\}$, the standard three terms and their complementary (negative) terms from Classical Syllogistics. One can write all the 32 pairs of categorical premises of the conclusive syllogisms with indefinite terms of types Barbara, Darapti, Darii and Disamis as, respectively, $\mathrm{A}\left(\mathrm{M}^{*}, \mathrm{P}^{*}\right) \mathrm{A}\left(\mathrm{S}^{*}, \mathrm{M}^{*}\right), \quad \mathrm{A}\left(\mathrm{M}^{*}, \mathrm{P}^{*}\right) \mathrm{A}\left(\mathrm{M}^{*}, \mathrm{~S}^{*}\right), \quad \mathrm{A}\left(\mathrm{M}^{*}, \mathrm{P}^{*}\right) \mathrm{I}\left(\mathrm{M}^{*}, \mathrm{~S}^{*}\right), \quad \mathrm{I}\left(\mathrm{M}^{*}, \mathrm{P}^{*}\right)$ $\mathrm{A}\left(\mathrm{M}^{*}, \mathrm{~S}^{*}\right)$. Their logical consequences (LCs) or conclusions are found by writing the term which is the "subject of the LC" as a union of the $2^{3}$ partitioning subsets of the universe of discourse, U. Felicitously, any logical consequence of a syllogism pinpoints a unique partitioning subset of $U$, and so does any conclusion of an n-term biliteral sorite. (U will then have $2^{n}$ partitioning subsets.) E.g., denoting intersections of terms (or sets) by juxtapositions and union of sets by plus signs, from the firstly listed Barbara's type PCPs, one gets two conclusions per pair of premises: $S^{*}=S^{*} M^{*}+S^{*} M^{*}=S^{*} M^{*}=S^{*} M^{*} P^{*}+S^{*} M * P^{* \prime}=S * M * P^{*}$; analogously $\mathrm{P}^{* \prime}=\mathrm{S}^{*} \mathrm{M}{ }^{*} \mathrm{P} *^{*}$. From these precise, "one partitioning subset of $\mathrm{U}$ " conclusions, one may eliminate the middle term $\mathrm{M}^{*}$ to find the standard Classical Syllogistics' conclusion: $\mathrm{A}\left(\mathrm{S}^{*}, \mathrm{P}^{*}\right)=\mathrm{A}\left(\mathrm{P}^{* \prime}, \mathrm{S}^{* \prime}\right)=\mathrm{E}\left(\mathrm{S}^{*}, \mathrm{P}^{* \prime}\right)$. Other types of conclusive syllogisms are treated similarly. Each of the eight syllogisms with indefinite terms of type Barbara corresponds to a chain inclusion $S^{*} \subseteq M^{*} \subseteq \mathrm{P}^{*}$. Analogously, all the eight syllogisms of type Darapti express the Darapti type inclusions: $\mathrm{M}^{*} \subseteq \mathrm{S}^{*}, \mathrm{M}^{*} \subseteq \mathrm{P}^{*}$. Both Darii and Disamis types are reducible to Darii's pattern: "The middle term intersects one other term and is included in the third one".
\end{abstract}

Keywords: Categorical Premises, Conclusive Syllogisms, Term Relabelings, Indefinite Terms.

\section{Introduction}

Existential Import will be shortened to ei, pair of categorical premises to PCP, logical consequence or conclusion to LC, and "partitioning subset of the universe of discourse U" will be shortened to subset of $\mathrm{U}$. One will distinguish between the premises' patterns, (which can be described in words and to which, in the case of a biliteral 
sorite with $\mathrm{n}$ terms following a most general pattern, should correspond $\mathrm{n}$ ! independent term orderings), and the premises' type - which refers to premises of a definite pattern and a particular term ordering, which can be collected under a single formula -using the "star notation", (already mentioned in the abstract), and thus takes care of all the $2^{\mathrm{n}}$ ways in which the terms or their complements can be used within that pattern and term ordering. For example, in the case of three terms, the Barbara's PCP pattern, can be described, in words, as a chain of two inclusions - which amounts to having two universal premises, one acting on the middle term $\mathrm{M}$ and the other one acting on $\mathrm{M}^{\prime}$ - the non-M, negative, or complementary term of $\mathrm{M}$ in the universal set U. (For more than three terms, the Barbara pattern can be described as an uninterrupted chain of inclusions.) Given three terms, to the Barbara's pattern correspond six permutations: three choices for the term which will play the role of the middle term, times two other choices for which term will be denoted by $\mathrm{P}-$ and, by convention, appear in the firstly listed premise - and which one will be denoted by S. For each of the six assignments of the M, P, S roles to the three given terms, the PCP set of eight Barbara's type conclusive syllogisms which one can build, can be represented as $S^{*} \subseteq M^{*} \subseteq P^{*}$, where $P^{*} \in\left\{P, P^{\prime}\right\}, M^{*} \in\left\{M, M^{\prime}\right\}, S^{*} \in\left\{S, S^{\prime}\right\}$. Since $S^{*} \subseteq M^{*} \subseteq P^{*}$ is equivalent to $\mathrm{P}^{*} \subseteq \mathrm{M}^{*} \subseteq \mathrm{S}^{*}$ the six permutations or orderings of the three terms are reduced to only three - the three choices for which term will play the role of the middle term. In general, for an $n$ terms Barbara type bilateral sorite, only $n ! / 2$ term permutations seem to be independent: the formula checks for $n=2$, (since $S^{*} \subseteq P^{*}$ is equivalent to $\left.\mathrm{P}^{*} \subseteq S^{*}\right)$, and $\mathrm{n}=3$. Supposing it is true for $\mathrm{n}$, when adding the $(\mathrm{n}+1)$ term, inserting it between the previous $n$ terms of each of the $n ! / 2$ independent permutations, should generate $(n+1)$ new independent permutations, thus giving $(n+1) ! / 2$ permutations for $(\mathrm{n}+1)$ terms. Obviously, to the Darapti pattern - one term out of the $\mathrm{n}$ terms is included in each of the other $\mathrm{n}-1$ terms - correspond not $\mathrm{n}$ ! independent permutations of the $\mathrm{n}$ terms, but only $\mathrm{n}$ choices of which term is to be included in each one of the other $\mathrm{n}-1$ terms. This is why, as for the Barbara type syllogisms, once the middle term is chosen, there are only $2^{3}=8$ different syllogisms of Darapti's type. For Darii's type syllogisms, all the 3 ! term permutations are independent. Thus, even after the middle term is chosen, a $\mathrm{P} \leftrightarrow \mathrm{S}$ permutation will distinguish between the eight Darii type syllogisms and the eight Disamis type syllogisms, raising to 32 the total number of valid syllogisms with indefinite terms. Interestingly, these 32 valid syllogisms with indefinite terms were described in about the same way by Reichenbach [1], who cites earlier authors, Churchman [2] and Smith [3], who systematically considered syllogisms with indefinite terms. See also Pratt [4] where I saw the Reichenbach reference. Alvarez and Correia [5] is another example of an extension of classical syllogisms to syllogisms with indefinite terms.

For the eight Barbara type syllogisms with indefinite terms, one can write down the single formula containing their PCPs and, listed after a column sign and separated by semi-columns, their entailed LCs (deduced as shown in the Abstract, using Jevons [8] method of decomposition of a set into partitioning subsets of $\mathrm{U}$. Jevons method is easier to apply if the universal premises are written as E statements instead of A statements.): 
(1) (Type Barbara) $\mathrm{A}\left(\mathrm{M}^{*}, \mathrm{P}^{*}\right) \mathrm{A}\left(\mathrm{S}^{*}, \mathrm{M}^{*}\right)=\mathrm{E}\left(\mathrm{M}^{*}, \mathrm{P}^{*}\right) \mathrm{E}\left(\mathrm{M}^{*}, \mathrm{~S}^{*}\right): \mathrm{S}^{*}=\mathrm{S}^{*} \mathrm{M}^{*} \mathrm{P}^{*}, \mathrm{P}^{*}=$ $\mathrm{P}^{* \prime} \mathrm{M}^{*} \mathrm{~S}^{* \prime} ; \mathrm{A}\left(\mathrm{S}^{*}, \mathrm{P}^{*}\right)\left[=\mathrm{A}\left(\mathrm{P}^{* \prime}, \mathrm{S}^{* \prime}\right)=\mathrm{E}\left(\mathrm{S}^{*}, \mathrm{P}^{*}\right)\right]$ - after $\mathrm{M}^{*}$ is dropped; $\mathrm{I}\left(\mathrm{S}^{*}, \mathrm{P}^{*}\right)$ - after ei on $\mathrm{S}^{*} ; \mathrm{I}\left(\mathrm{P}^{*}, \mathrm{~S}^{* \prime}\right)$ - after ei on $\mathrm{P}^{*}$.'

As mentioned, given $\mathrm{n}$ terms, when using a "most general pattern" for valid syllogisms, one may construct, out of the $\mathrm{n}$ terms, a valid $\mathrm{n}$-term syllogistic argument or sorite in $\mathrm{n} ! 2^{\mathrm{n}}$ ways, (some of these valid arguments might be sound), since the order in which the terms appear in the argument matters, and it also matters if the term, or, instead, its complement, appears in the argument. To a "most general pattern" correspond $\mathrm{n}$ ! permutations of the terms satisfying the pattern. Once that ordering is decided, using the above "star notation" for a term and its complement in U, one can collect into a single formula both the PCPs and their LCs. The above formula (1) encompasses, (when the middle term was already agreed upon), all the conclusive syllogisms and their conclusions (LCs) for the Barbara pattern of conclusive syllogisms. The next Section gives formulas for three other types of conclusive syllogisms: Darapti, Darii and Disamis. They correspond to just two patterns: Darapti and Darii (the latter pattern might have been called the Disamis pattern as well). Together with the formula (1) for Barbara type conclusive syllogisms, these formulas represent an extension of the Classical Syllogistics to premises and LCs which contain negative terms - thus forgoing the syllogistic figures. For valid syllogisms, the Classical Syllogistics accepts, by definition, only these LC formats: A(S,P), E(S,P), I(S,P), O(S,P). By contrast, even after eliminating the middle term from it, the LC of a conclusive syllogism can have any of the eight formats $\mathrm{A}\left(\mathrm{S}^{*}, \mathrm{P}^{*}\right), \mathrm{I}\left(\mathrm{S}^{*}, \mathrm{P}^{*}\right)$, where $\mathrm{P}^{*} \in\left\{\mathrm{P}, \mathrm{P}^{\prime}\right\}$, $S^{*} \in\left\{S, S^{\prime}\right\}$. The next Section also lists the patterns and formulas for the 32 PCPs which do not entail any LCs. All the 64 possible PCPs are elements of an eight by eight PCP matrix generated by pairing-up the eight categorical P-premises, $\mathrm{A}\left(\mathrm{M}^{*}, \mathrm{P}^{*}\right)$, (or $\mathrm{E}\left(\mathrm{M}^{*}, \mathrm{P}^{*}\right)$ ), $\mathrm{I}\left(\mathrm{M}^{*}, \mathrm{P}^{*}\right)$, with the eight categorical $\mathrm{S}$-premises, $\mathrm{A}\left(\mathrm{M}^{*}, \mathrm{~S}^{*}\right)$, (or $\left.\mathrm{E}\left(\mathrm{M}^{*}, \mathrm{~S}^{*}\right)\right), \mathrm{I}\left(\mathrm{M}^{*}, \mathrm{~S}^{*}\right)$, where the premises contain indefinite (both positive and negative) terms: $\mathrm{M}^{*} \in\left\{\mathrm{M}, \mathrm{M}^{\prime}\right\}, \mathrm{P}^{*} \in\left\{\mathrm{P}, \mathrm{P}^{\prime}\right\}, \mathrm{S}^{*} \in\left\{\mathrm{S}, \mathrm{S}^{\prime}\right\}$. Interestingly, the $64 \mathrm{PCP}$ elements of the above matrix can be split into eight groups of eight matrix elements each, the eight PCPs in the same group representing - one PCP per type - all the eight types of PCPs (conclusive or not), which correspond to all the five PCP patterns - three conclusive patterns plus two patterns for the PCPs which do not entail any LCs. One of the latter two patterns corresponds to pairs of particular premises, the other pattern corresponds to one particular premise acting on $\mathrm{M}^{*}$ and another universal premise acting on $\mathrm{M}^{*}$. A transition between any two of the eight groups of eight PCPs each denote them by $\mathrm{H}_{1}, \mathrm{H}_{2}, \ldots, \mathrm{H}_{8}$ - is made via a transformation from a group $\mathrm{G}$ with eight elements, which, by definition, acts on the triplets $\left(\mathrm{M}^{*}, \mathrm{P}^{*}, \mathrm{~S}^{*}\right)$ in the following way: $\mathrm{s}\left(\mathrm{M}^{*}, \mathrm{P}^{*}, \mathrm{~S}^{*}\right)=\left(\mathrm{M}^{*}, \mathrm{P}^{*}, \mathrm{~S}^{*}\right), \mathrm{p}\left(\mathrm{M}^{*}, \mathrm{P}^{*}, \mathrm{~S}^{*}\right)=\left(\mathrm{M}^{*}, \mathrm{P}^{*}, \mathrm{~S}^{*}\right), \mathrm{m}\left(\mathrm{M}^{*}, \mathrm{P}^{*}\right.$, $\left.\mathrm{S}^{*}\right)=\left(\mathrm{M}^{*}, \mathrm{P}^{*}, \mathrm{~S}^{*}\right), \mathrm{mp}\left(\mathrm{M}^{*}, \mathrm{P}^{*}, \mathrm{~S}^{*}\right)=\left(\mathrm{M}^{*}, \mathrm{P}^{*}, \mathrm{~S}^{*}\right), \mathrm{ms}\left(\mathrm{M}^{*}, \mathrm{P}^{*}, \mathrm{~S}^{*}\right)=\left(\mathrm{M}^{*}, \mathrm{P}^{*}, \mathrm{~S}^{* \prime}\right)$, $\operatorname{ps}\left(\mathrm{M}^{*}, \mathrm{P}^{*}, \mathrm{~S}^{*}\right)=\left(\mathrm{M}^{*}, \mathrm{P}^{* \prime}, \mathrm{S}^{*}\right), \mathrm{msp}\left(\mathrm{M}^{*}, \mathrm{P}^{*}, \mathrm{~S}^{*}\right)=\left(\mathrm{M}^{* \prime}, \mathrm{P}^{* \prime}, \mathrm{S}^{* \prime}\right), \mathrm{e}\left(\mathrm{M}^{*}, \mathrm{P}^{*}\right.$, $\left.\mathrm{S}^{*}\right)=\left(\mathrm{M}^{*}, \mathrm{P}^{*}, \mathrm{~S}^{*}\right)$. The action of $\mathrm{G}$ on $\mathrm{H}_{1}, \mathrm{H}_{2}, \ldots, \mathrm{H}_{8}$ will be displayed in Section 2. 


\section{The PCPs and LCs of the 32 conclusive syllogisms with indefinite terms and the 32 PCPs which do not entail any LC}

Here are the formulas for the other conclusive PCPs and, after the column sign and separated by semi-columns, all the LCs, given in both the precise - "one subset of U" format, and also given in the Classical Syllogistics format - the one which does not contain the middle term.

One uses again Jevons' [6] method of decomposing the set of interest into a union of subsets of $U$, to find that each of the eight Darapti type PCPs, $A\left(M^{*}, P^{*}\right) A\left(M^{*}, S^{*}\right)$, entails just one universal $L C: M^{*}=M^{*} P^{*}+M^{*} P^{*}=M^{*} P^{*}=M^{*} P^{*} S^{*}+M^{*} P^{*} S^{* 1}$ $=\mathrm{M}^{*} \mathrm{P}^{*} \mathrm{~S}^{*}$, or $\mathrm{A}\left(\mathrm{M}^{*}, \mathrm{P}^{*} \mathrm{~S}^{*}\right)$ which reflects the inclusions $\mathrm{M}^{*} \subseteq \mathrm{P}^{*}$ and $\mathrm{M}^{*} \subseteq \mathrm{S}^{*}$ asserted by the premises. Thus, for the type Darapti PCPs, the middle term itself is "the subject" of the precise LC; it can be eliminated only via ei on $\mathrm{M}^{*}$ :

(2) (Type Darapti) A(M*,P*)A(M*,S*): $M^{*}=M^{*} \mathrm{P}^{*} \mathrm{~S}^{*}, \mathrm{~A}\left(\mathrm{M}^{*}, \mathrm{P}^{*} \mathrm{~S}^{*}\right) ; \mathrm{M}^{*} \mathrm{P}^{*} \mathrm{~S}^{*} \neq \varnothing$ if $\mathrm{M}^{*} \neq \varnothing$, or, $\mathrm{I}\left(\mathrm{S}^{*}, \mathrm{P}^{*}\right)$ if $\mathrm{M}^{*} \neq \varnothing$ and after $\mathrm{M}^{*}$ is eliminated.

There are eight distinct PCPs of type Darii, $A\left(M^{*}, \mathrm{P}^{*}\right) \mathrm{I}\left(\mathrm{M}^{*}, \mathrm{~S}^{*}\right)$, and eight distinct PCPs of type Disamis, I( $\left.\mathrm{M}^{*}, \mathrm{P}^{*}\right) \mathrm{A}\left(\mathrm{M}^{*}, \mathrm{~S}^{*}\right)$. The LCs are obtained via the decompositions: $\varnothing \neq M * S *=M * S * P^{*}+M^{*} S * P^{*}=M^{*} S^{*} P^{*}, \quad$ and, $\quad$ resp., $\varnothing \neq M * P^{*}=M * S * P^{*}+$ $\mathrm{M} * \mathrm{~S} * \mathrm{P} *=\mathrm{M} * \mathrm{~S} * \mathrm{P} *$ Thus:

(3i) (Type Darii) A(M*,P*)I(M*,S*): $\mathrm{M}^{*} \mathrm{~S}^{*} \mathrm{P}^{*} \neq \varnothing ; \mathrm{I}\left(\mathrm{S}^{*}, \mathrm{P}^{*}\right)\left[=\mathrm{O}\left(\mathrm{S}^{*}, \mathrm{P}^{*}\right)\right]-$ after $\mathrm{M}^{*}$ is dropped from the LC.

(3ii) (Type Disamis) I( $\left.\mathrm{M}^{*}, \mathrm{P}^{*}\right) \mathrm{A}\left(\mathrm{M}^{*}, \mathrm{~S}^{*}\right): \mathrm{M}^{*} \mathrm{P}^{*} \mathrm{~S} \neq \varnothing ; \mathrm{I}\left(\mathrm{P}^{*}, \mathrm{~S}^{*}\right)\left[=\mathrm{O}\left(\mathrm{S}^{*}, \mathrm{P}^{*}\right)\right]-$ after $\mathrm{M}^{*}$ is dropped from the LC.

Note that the Barbara and Darapti patterns and formulas are invariant under the permutation $\mathrm{P} \leftrightarrow \mathrm{S}$, but Darii's pattern and formula is not - this is why one has conclusive syllogisms of both Darii and Disamis types.

Finally, one lists the formulas corresponding to the two patterns and four types of PCPs which do not entail any LCs. To the two particular premises pattern correspond the formulas:

(4i) $\mathrm{I}\left(\mathrm{M}^{*}, \mathrm{P}^{*}\right) \mathrm{I}\left(\mathrm{M}^{*}, \mathrm{~S}^{*}\right)$,

(4ii) $\mathrm{I}\left(\mathrm{M}^{*}, \mathrm{P}^{*}\right) \mathrm{I}\left(\mathrm{M}^{*}, \mathrm{~S}^{*}\right)$.

To the pattern of one universal premise plus one particular premise, one "acting" on $\mathrm{M}$ and the other on $\mathrm{M}^{\prime}$ correspond the formulas:

(5i) $\mathrm{A}\left(\mathrm{P}^{*}, \mathrm{M}^{*}\right) \mathrm{I}\left(\mathrm{M}^{*}, \mathrm{~S}^{*}\right)$,

(5ii) $\mathrm{I}\left(\mathrm{M}^{*}, \mathrm{P}^{*}\right) \mathrm{A}\left(\mathrm{S}^{*}, \mathrm{M}^{*}\right)$.

The above eight formulas, each covering eight PCPs, exhaust all the elements of the 64 element PCP matrix. One can now establish a function, by mapping each of the eight triplets $\left(\mathrm{M}^{*}, \mathrm{P}^{*}, \mathrm{~S}^{*}\right)$, to the set of eight corresponding conclusive syllogisms and PCPs obtained when substituting the values of that triplet into the formulas (1)-(5ii). Each of these "eight PCPs sets", $\mathrm{H}_{1}, \mathrm{H}_{2}, \ldots, \mathrm{H}_{8}$, corresponds to a set of values for the term variables $\left(S^{*}, P^{*}, M^{*}\right)$, where $S^{*} \in\left\{S, S^{\prime}\right\}, P^{*} \in\left\{P, P^{\prime}\right\}, M^{*} \in\left\{M, M^{\prime}\right\}$. This assures that there is a unique transformation from the group $G$ mapping any of the sets of eight PCPs and their generated LCs, (if any), $\mathrm{H}_{1}, \mathrm{H}_{2}, \ldots, \mathrm{H}_{8}$, into another such set of eight PCPs and their respective LCs, (if any). Listing on one column the four conclu- 
sive PCPs, and on the second column their LCs, and listing on one row all the four non-conclusive PCPs, the sets, $\mathrm{H}_{1}, \mathrm{H}_{2}, \ldots, \mathrm{H}_{8}$, of eight PCPs each, and their entailed LCs - if any, are:

$\mathbf{H}_{1}$ :

Conclusive syllogisms for $\mathrm{M}^{*}=\mathrm{M}, \mathrm{S}^{*}=\mathrm{S}, \mathrm{P}^{*}=\mathrm{P}$ :

$\mathrm{A}(\mathrm{M}, \mathrm{P}) \mathrm{A}(\mathrm{S}, \mathrm{M}) \quad \mathrm{S}=\mathrm{SPM}, \quad \mathrm{P}^{\prime}=\mathrm{S}^{\prime} \mathrm{P}^{\prime} \mathrm{M}^{\prime}, \quad \mathrm{A}(\mathrm{S}, \mathrm{P})=\mathrm{A}\left(\mathrm{P}^{\prime}, \mathrm{S}^{\prime}\right), \quad$ Barbara $\mathrm{I}(\mathrm{S}, \mathrm{P})$ if $\mathrm{S} \neq \varnothing$, Barbari; $\mathrm{I}\left(\mathrm{P}^{\prime}, \mathrm{S}^{\prime}\right)$ if $\mathrm{P}^{\prime} \neq \varnothing$, No name

$\mathrm{A}(\mathrm{M}, \mathrm{P}) \mathrm{A}(\mathrm{M}, \mathrm{S}) \quad \mathrm{M}=\mathrm{SPM}$ the non-ei Darapti. If $\mathrm{M} \neq \varnothing$ : I(S,P), the ei Darapti

$\mathrm{A}(\mathrm{M}, \mathrm{P}) \mathrm{I}(\mathrm{M}, \mathrm{S}) \quad \mathrm{SPM} \neq \varnothing \quad$ or $\quad \mathrm{I}(\mathrm{S}, \mathrm{P}), \quad$ Darii

$\mathrm{I}(\mathrm{M}, \mathrm{P}) \mathrm{A}(\mathrm{M}, \mathrm{S}) \quad \mathrm{SPM} \neq \varnothing \quad$ or $\quad \mathrm{I}(\mathrm{S}, \mathrm{P}), \quad$ Disamis

Non-conclusive PCPs for $\mathrm{M}^{*}=\mathrm{M}, \mathrm{S}^{*}=\mathrm{S}, \mathrm{P}^{*}=\mathrm{P}$ :

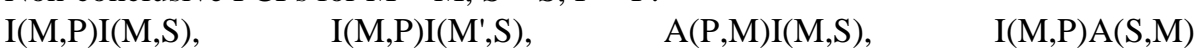

$\mathbf{H}_{2}$ :

Conclusive syllogisms for $\mathrm{M}^{*}=\mathrm{M}, \mathrm{S}^{*}=\mathrm{S}^{\prime}, \mathrm{P}^{*}=\mathrm{P}$ :

$\left.\mathrm{A}(\mathrm{M}, \mathrm{P}) \mathrm{A}\left(\mathrm{S}^{\prime}, \mathrm{M}\right) \quad \mathrm{S}^{\prime}=\mathrm{S}^{\prime} \mathrm{PM}, \quad \mathrm{P}^{\prime}=\mathrm{SP}^{\prime} \mathrm{M}^{\prime}, \mathrm{A}\left(\mathrm{S}^{\prime}, \mathrm{P}\right)\right)=\mathrm{A}\left(\mathrm{P}^{\prime}, \mathrm{S}\right), \quad$ No name

$\mathrm{I}\left(\mathrm{S}^{\prime}, \mathrm{P}\right)=\mathrm{O}(\mathrm{P}, \mathrm{S})$ if $\mathrm{S}^{\prime} \neq \varnothing, ; \mathrm{I}\left(\mathrm{P}^{\prime}, \mathrm{S}\right)=\mathrm{O}(\mathrm{S}, \mathrm{P})$ if $\mathrm{P}^{\prime} \neq \varnothing$, No name

$\mathrm{A}(\mathrm{M}, \mathrm{P}) \mathrm{A}\left(\mathrm{M}, \mathrm{S}^{\prime}\right) \quad \mathrm{M}=\mathrm{S}^{\prime} \mathrm{PM}$ no name. If $\mathrm{M} \neq \varnothing: \quad \mathrm{I}\left(\mathrm{S}^{\prime}, \mathrm{P}\right)$, No name

$\mathrm{A}(\mathrm{M}, \mathrm{P}) \mathrm{I}\left(\mathrm{M}, \mathrm{S}^{\prime}\right) \quad \mathrm{S}^{\prime} \mathrm{PM} \neq \varnothing \quad$ or $\quad \mathrm{I}\left(\mathrm{S}^{\prime}, \mathrm{P}\right), \quad$ No name

$\mathrm{I}(\mathrm{M}, \mathrm{P}) \mathrm{A}\left(\mathrm{M}, \mathrm{S}^{\prime}\right) \quad \mathrm{S}^{\prime} \mathrm{PM} \neq \varnothing \quad$ or $\quad \mathrm{I}\left(\mathrm{S}^{\prime}, \mathrm{P}\right), \quad$ No name

Non-conclusive PCPs for $\mathrm{M}^{*}=\mathrm{M}, \mathrm{S}^{*}=\mathrm{S}^{\prime}, \mathrm{P}^{*}=\mathrm{P}$ :

$\mathrm{I}(\mathrm{M}, \mathrm{P}) \mathrm{I}\left(\mathrm{M}, \mathrm{S}^{\prime}\right), \quad \mathrm{I}(\mathrm{M}, \mathrm{P}) \mathrm{I}\left(\mathrm{M}^{\prime}, \mathrm{S}^{\prime}\right), \quad \mathrm{A}(\mathrm{P}, \mathrm{M}) \mathrm{I}\left(\mathrm{M}, \mathrm{S}^{\prime}\right), \quad \mathrm{I}(\mathrm{M}, \mathrm{P}) \mathrm{A}\left(\mathrm{S}^{\prime}, \mathrm{M}\right)$

$\mathbf{H}_{3}$ :

Conclusive syllogisms for $\mathrm{M}^{*}=\mathrm{M}, \mathrm{S}^{*}=\mathrm{S}, \mathrm{P}^{*}=\mathrm{P}^{\prime}$ :

$\left.\mathrm{A}\left(\mathrm{M}, \mathrm{P}^{\prime}\right) \mathrm{A}(\mathrm{S}, \mathrm{M}) \quad \mathrm{S}=\mathrm{SP}{ }^{\prime} \mathrm{M}, \mathrm{P}=\mathrm{S}^{\prime} \mathrm{PM} \mathrm{M}^{\prime}, \mathrm{A}\left(\mathrm{S}, \mathrm{P}^{\prime}\right)\right)=\mathrm{A}\left(\mathrm{P}, \mathrm{S}^{\prime}\right)=\mathrm{E}(\mathrm{S}, \mathrm{P})$ Celarent/Cesare

$\mathrm{I}\left(\mathrm{S}, \mathrm{P}^{\prime}\right)=\mathrm{O}(\mathrm{S}, \mathrm{P})$ if $\mathrm{S} \neq \varnothing$, Celaront/Cesaro; $\mathrm{O}(\mathrm{P}, \mathrm{S})$ if $\mathrm{P}^{\prime} \neq \varnothing$, No name

$\mathrm{A}\left(\mathrm{M}, \mathrm{P}^{\prime}\right) \mathrm{A}(\mathrm{M}, \mathrm{S}) \quad \mathrm{M}=S \mathrm{P}^{\prime} \mathrm{M}$ non-ei Felapton. If $\mathrm{M} \neq \varnothing: \mathrm{O}(\mathrm{S}, \mathrm{P})$, the ei Felapton/Fesapo

$\mathrm{A}\left(\mathrm{M}, \mathrm{P}^{\prime}\right) \mathrm{I}(\mathrm{M}, \mathrm{S}) \quad \mathrm{S} \mathrm{P}^{\prime} \mathrm{M} \neq \varnothing$ or $\quad \mathrm{I}\left(\mathrm{S}, \mathrm{P}^{\prime}\right)=\mathrm{O}(\mathrm{S}, \mathrm{P})$, Ferio/Festino/Ferison/Fresison $\mathrm{I}\left(\mathrm{M}, \mathrm{P}^{\prime}\right) \mathrm{A}(\mathrm{M}, \mathrm{S}) \quad \mathrm{SP} \mathrm{M} \neq \varnothing \quad$ or $\left.\quad \mathrm{I}\left(\mathrm{S}, \mathrm{P}^{\prime}\right)\right)=\mathrm{O}(\mathrm{S}, \mathrm{P}), \quad$ Bocardo

Non-conclusive PCPs for $\mathrm{M}^{*}=\mathrm{M}, \mathrm{S}^{*}=\mathrm{S}, \mathrm{P}^{*}=\mathrm{P}^{\prime}$ :

$\mathrm{I}\left(\mathrm{M}, \mathrm{P}^{\prime}\right) \mathrm{I}(\mathrm{M}, \mathrm{S}), \quad \mathrm{I}\left(\mathrm{M}, \mathrm{P}^{\prime}\right) \mathrm{I}\left(\mathrm{M}^{\prime}, \mathrm{S}\right), \quad \mathrm{A}\left(\mathrm{P}^{\prime}, \mathrm{M}\right) \mathrm{I}(\mathrm{M}, \mathrm{S}), \quad \mathrm{I}\left(\mathrm{M}, \mathrm{P}^{\prime}\right) \mathrm{A}(\mathrm{S}, \mathrm{M})$

$\mathbf{H}_{4}$ :

Conclusive syllogisms for $\mathrm{M}^{*}=\mathrm{M}, \mathrm{S}^{*}=\mathrm{S}^{\prime}, \mathrm{P}^{*}=\mathrm{P}^{\prime}$ :

$\left.\mathrm{A}\left(\mathrm{M}, \mathrm{P}^{\prime}\right) \mathrm{A}\left(\mathrm{S}^{\prime}, \mathrm{M}\right) \quad \mathrm{S}^{\prime}=\mathrm{S}^{\prime} \mathrm{P} \mathrm{P}^{\prime} \mathrm{M}, \quad \mathrm{P}=\mathrm{SPM}, \mathrm{A}\left(\mathrm{S}^{\prime}, \mathrm{P}^{\prime}\right)\right)=\mathrm{A}(\mathrm{P}, \mathrm{S}), \quad$ No name

$\mathrm{I}\left(\mathrm{S}^{\prime}, \mathrm{P}^{\prime}\right)$ if $\mathrm{S}^{\prime} \neq \varnothing, ; \mathrm{I}(\mathrm{P}, \mathrm{S})$ if $\mathrm{P} \neq \varnothing$, Bramantip' (the prime refers to $\mathrm{M}^{\prime}$ in $\left.\mathrm{P}=\mathrm{PSM} \mathrm{M}^{\prime}\right)$.

$\mathrm{A}\left(\mathrm{M}, \mathrm{P}^{\prime}\right) \mathrm{A}\left(\mathrm{M}, \mathrm{S}^{\prime}\right) \quad \mathrm{M}=\mathrm{S}^{\prime} \mathrm{P}^{\prime} \mathrm{M}$ no name. If $\mathrm{M} \neq \varnothing: \quad \mathrm{I}\left(\mathrm{S}^{\prime}, \mathrm{P}^{\prime}\right)$, No name

$\mathrm{A}\left(\mathrm{M}, \mathrm{P}^{\prime}\right) \mathrm{I}\left(\mathrm{M}, \mathrm{S}^{\prime}\right) \quad \mathrm{S}^{\prime} \mathrm{P}^{\prime} \mathrm{M} \neq \varnothing$ or $\quad \mathrm{I}\left(\mathrm{S}^{\prime}, \mathrm{P}^{\prime}\right), \quad$ No name

$\mathrm{I}\left(\mathrm{M}, \mathrm{P}^{\prime}\right) \mathrm{A}\left(\mathrm{M}, \mathrm{S}^{\prime}\right) \quad \mathrm{S}^{\prime} \mathrm{P}^{\prime} \mathrm{M} \neq \varnothing \quad$ or $\quad \mathrm{I}\left(\mathrm{S}^{\prime}, \mathrm{P}^{\prime}\right), \quad$ No name

Non-conclusive PCPs for $\mathrm{M}^{*}=\mathrm{M}, \mathrm{S}^{*}=\mathrm{S}^{\prime}, \mathrm{P}^{*}=\mathrm{P}^{\prime}$ :

$\mathrm{I}\left(\mathrm{M}, \mathrm{P}^{\prime}\right) \mathrm{I}\left(\mathrm{M}, \mathrm{S}^{\prime}\right), \quad \mathrm{I}\left(\mathrm{M}, \mathrm{P}^{\prime}\right) \mathrm{I}\left(\mathrm{M}^{\prime}, \mathrm{S}^{\prime}\right), \quad \mathrm{A}\left(\mathrm{P}^{\prime}, \mathrm{M}\right) \mathrm{I}\left(\mathrm{M}, \mathrm{S}^{\prime}\right), \quad \mathrm{I}\left(\mathrm{M}, \mathrm{P}^{\prime}\right) \mathrm{A}\left(\mathrm{S}^{\prime}, \mathrm{M}\right)$ 
$\mathbf{H}_{5}$ :

Conclusive syllogisms for $\mathrm{M}^{*}=\mathrm{M}^{\prime}, \mathrm{S}^{*}=\mathrm{S}, \mathrm{P}^{*}=\mathrm{P}$ :

$\mathrm{A}\left(\mathrm{M}^{\prime}, \mathrm{P}\right) \mathrm{A}\left(\mathrm{S}, \mathrm{M}^{\prime}\right) \quad \mathrm{S}=\mathrm{SPM}, \quad \mathrm{P}^{\prime}=\mathrm{S}^{\prime} \mathrm{P}^{\prime} \mathrm{M}, \quad \mathrm{A}(\mathrm{S}, \mathrm{P})=\mathrm{A}\left(\mathrm{P}^{\prime}, \mathrm{S}^{\prime}\right), \quad$ Barbara${ }^{\prime}$ $\mathrm{I}(\mathrm{S}, \mathrm{P})$ if $\mathrm{S} \neq \varnothing$, Barbari'; $\mathrm{I}\left(\mathrm{P}^{\prime}, \mathrm{S}^{\prime}\right)$ if $\mathrm{P}^{\prime} \neq \varnothing$, No name $\mathrm{A}\left(\mathrm{M}^{\prime}, \mathrm{P}\right) \mathrm{A}\left(\mathrm{M}^{\prime}, \mathrm{S}\right) \quad \mathrm{M}^{\prime}=\mathrm{SPM}$ the non-ei Darapti'. If $\mathrm{M} \neq \varnothing$ : I(S,P), the ei Darapti' $\mathrm{A}\left(\mathrm{M}^{\prime}, \mathrm{P}\right) \mathrm{I}\left(\mathrm{M}^{\prime}, \mathrm{S}\right) \quad \mathrm{SPM}^{\prime} \neq \varnothing \quad$ or $\quad \mathrm{I}(\mathrm{S}, \mathrm{P}), \quad$ Darii' $\mathrm{I}\left(\mathrm{M}^{\prime}, \mathrm{P}\right) \mathrm{A}\left(\mathrm{M}^{\prime}, \mathrm{S}\right) \quad \mathrm{SPM} \neq \varnothing \quad$ or $\quad \mathrm{I}(\mathrm{S}, \mathrm{P}), \quad$ Disamis'/Dimaris' Non-conclusive PCPs for $\mathrm{M}^{*}=\mathrm{M}, \mathrm{S}^{*}=\mathrm{S}, \mathrm{P}^{*}=\mathrm{P}$ :

$\mathrm{I}\left(\mathrm{M}^{\prime}, \mathrm{P}\right) \mathrm{I}\left(\mathrm{M}^{\prime}, \mathrm{S}\right), \quad \mathrm{I}\left(\mathrm{M}^{\prime}, \mathrm{P}\right) \mathrm{I}(\mathrm{M}, \mathrm{S}), \quad \mathrm{A}\left(\mathrm{P}, \mathrm{M}^{\prime}\right) \mathrm{I}\left(\mathrm{M}^{\prime}, \mathrm{S}\right), \quad \mathrm{I}\left(\mathrm{M}^{\prime}, \mathrm{P}\right) \mathrm{A}\left(\mathrm{S}, \mathrm{M}^{\prime}\right)$

$\mathbf{H}_{6}$ :

Conclusive syllogisms for $\mathrm{M}^{*}=\mathrm{M}^{\prime}, \mathrm{S}^{*}=\mathrm{S}^{\prime}, \mathrm{P}^{*}=\mathrm{P}$ :

$\left.\mathrm{A}\left(\mathrm{M}^{\prime}, \mathrm{P}\right) \mathrm{A}\left(\mathrm{S}^{\prime}, \mathrm{M}^{\prime}\right) \quad \mathrm{S}^{\prime}=\mathrm{S}^{\prime} \mathrm{PM}, \quad \mathrm{P}^{\prime}=\mathrm{SP} \mathrm{P}^{\prime} \mathrm{M}, \mathrm{A}\left(\mathrm{S}^{\prime}, \mathrm{P}\right)\right)=\mathrm{A}\left(\mathrm{P}^{\prime}, \mathrm{S}\right), \quad$ No name $\mathrm{I}\left(\mathrm{S}^{\prime}, \mathrm{P}\right)=\mathrm{O}(\mathrm{P}, \mathrm{S})$ if $\mathrm{S}^{\prime} \neq \varnothing, ; \mathrm{I}\left(\mathrm{P}^{\prime}, \mathrm{S}\right)=\mathrm{O}(\mathrm{S}, \mathrm{P})$ if $\mathrm{P}^{\prime} \neq \varnothing$, No name $\mathrm{A}\left(\mathrm{M}^{\prime}, \mathrm{P}\right) \mathrm{A}\left(\mathrm{M}^{\prime}, \mathrm{S}^{\prime}\right) \quad \mathrm{M}^{\prime}=\mathrm{S}^{\prime} \mathrm{PM} \quad$ no name. If $\mathrm{M}^{\prime} \neq \varnothing: \quad \mathrm{I}\left(\mathrm{S}^{\prime}, \mathrm{P}\right)$, No name $\mathrm{A}\left(\mathrm{M}^{\prime}, \mathrm{P}\right) \mathrm{I}\left(\mathrm{M}^{\prime}, \mathrm{S}^{\prime}\right) \quad \mathrm{S}^{\prime} \mathrm{PM} \neq \varnothing \quad$ or $\quad \mathrm{I}\left(\mathrm{S}^{\prime}, \mathrm{P}\right), \quad$ No name $\mathrm{I}\left(\mathrm{M}^{\prime}, \mathrm{P}\right) \mathrm{A}\left(\mathrm{M}^{\prime}, \mathrm{S}^{\prime}\right) \quad \mathrm{S}^{\prime} \mathrm{PM} \neq \varnothing \quad$ or $\quad \mathrm{I}\left(\mathrm{S}^{\prime}, \mathrm{P}\right), \quad$ No name Non-conclusive PCPs for $\mathrm{M}^{*}=\mathrm{M}^{\prime}, \mathrm{S}^{*}=\mathrm{S}^{\prime}, \mathrm{P}^{*}=\mathrm{P}$ :

$\mathrm{I}\left(\mathrm{M}^{\prime}, \mathrm{P}\right) \mathrm{I}\left(\mathrm{M}^{\prime}, \mathrm{S}^{\prime}\right), \quad \mathrm{I}\left(\mathrm{M}^{\prime}, \mathrm{P}\right) \mathrm{I}\left(\mathrm{M}, \mathrm{S}^{\prime}\right), \quad \mathrm{A}\left(\mathrm{P}, \mathrm{M}^{\prime}\right) \mathrm{I}\left(\mathrm{M}^{\prime}, \mathrm{S}^{\prime}\right), \quad \mathrm{I}\left(\mathrm{M}^{\prime}, \mathrm{P}\right) \mathrm{A}\left(\mathrm{S}^{\prime}, \mathrm{M}^{\prime}\right)$

$\mathbf{H}_{7}$ :

Conclusive syllogisms for $\mathrm{M}^{*}=\mathrm{M}^{\prime}, \mathrm{S}^{*}=\mathrm{S}, \mathrm{P}^{*}=\mathrm{P}^{\prime}$ :

$\left.\mathrm{A}\left(\mathrm{M}^{\prime}, \mathrm{P}^{\prime}\right) \mathrm{A}\left(\mathrm{S}, \mathrm{M}^{\prime}\right) \quad \mathrm{S}=\mathrm{SP} \mathrm{P}^{\prime} \mathrm{M}^{\prime}, \mathrm{P}=\mathrm{S} \mathrm{P}^{\prime} \mathrm{M}, \mathrm{A}\left(\mathrm{S}, \mathrm{P}^{\prime}\right)\right)=\mathrm{A}\left(\mathrm{P}, \mathrm{S}^{\prime}\right)=\mathrm{E}(\mathrm{S}, \mathrm{P}) \quad$ Camestres/ Camenes or Celarent'/Cesare'; $\quad \mathrm{I}\left(\mathrm{S}, \mathrm{P}^{\prime}\right)=\mathrm{O}(\mathrm{S}, \mathrm{P})$ if $\mathrm{S} \neq \varnothing$,

Camestros/Camenos; $\mathrm{O}(\mathrm{P}, \mathrm{S})$ if $\mathrm{P} \neq \varnothing, \quad$ No name

$\mathrm{A}\left(\mathrm{M}^{\prime}, \mathrm{P}^{\prime}\right) \mathrm{A}\left(\mathrm{M}^{\prime}, \mathrm{S}\right) \quad \mathrm{M}^{\prime}=\mathrm{SP}^{\prime} \mathrm{M}^{\prime}$ non-ei Felapton'. If $\mathrm{M} \neq \varnothing: \mathrm{O}(\mathrm{S}, \mathrm{P})$, the ei Felapton'/Fesapo'

$\mathrm{A}\left(\mathrm{M}^{\prime}, \mathrm{P}^{\prime}\right) \mathrm{I}\left(\mathrm{M}^{\prime}, \mathrm{S}\right) \quad \mathrm{SP}^{\prime} \mathrm{M}^{\prime} \neq \varnothing$ or $\mathrm{I}\left(\mathrm{S}, \mathrm{P}^{\prime}\right)=\mathrm{O}(\mathrm{S}, \mathrm{P})$, Baroco or $\quad$ Ferio'/Festino'/

Ferison'/Fresison'

$\mathrm{I}\left(\mathrm{M}^{\prime}, \mathrm{P}^{\prime}\right) \mathrm{A}\left(\mathrm{M}^{\prime}, \mathrm{S}\right) \quad \mathrm{SP}^{\prime} \mathrm{M}^{\prime} \neq \varnothing \quad$ or $\left.\quad \mathrm{I}\left(\mathrm{S}, \mathrm{P}^{\prime}\right)\right)=\mathrm{O}(\mathrm{S}, \mathrm{P}), \quad$ Bocardo'

Non-conclusive PCPs for $\mathrm{M}^{*}=\mathrm{M}^{\prime}, \mathrm{S}^{*}=\mathrm{S}, \mathrm{P}^{*}=\mathrm{P}^{\prime}$ :

$\mathrm{I}\left(\mathrm{M}^{\prime}, \mathrm{P}^{\prime}\right) \mathrm{I}\left(\mathrm{M}^{\prime}, \mathrm{S}\right), \quad \mathrm{I}\left(\mathrm{M}^{\prime}, \mathrm{P}^{\prime}\right) \mathrm{I}(\mathrm{M}, \mathrm{S}), \quad \mathrm{A}\left(\mathrm{P}^{\prime}, \mathrm{M}^{\prime}\right) \mathrm{I}\left(\mathrm{M}^{\prime}, \mathrm{S}\right), \quad \mathrm{I}\left(\mathrm{M}^{\prime}, \mathrm{P}^{\prime}\right) \mathrm{A}\left(\mathrm{S}, \mathrm{M}^{\prime}\right)$

$\mathbf{H}_{\mathbf{8}}$ :

Conclusive syllogisms for $\mathrm{M}^{*}=\mathrm{M}^{\prime}, \mathrm{S}^{*}=\mathrm{S}^{\prime}, \mathrm{P}^{*}=\mathrm{P}^{\prime}$ :

$\mathrm{A}\left(\mathrm{M}^{\prime}, \mathrm{P}^{\prime}\right) \mathrm{A}\left(\mathrm{S}^{\prime}, \mathrm{M}^{\prime}\right) \quad \mathrm{S}^{\prime}=\mathrm{S}^{\prime} \mathrm{P}^{\prime} \mathrm{M}^{\prime}, \quad \mathrm{P}=\mathrm{SPM}, \mathrm{A}\left(\mathrm{S}^{\prime}, \mathrm{P}^{\prime}\right)=\mathrm{A}(\mathrm{P}, \mathrm{S}), \quad$ No name $\mathrm{I}\left(\mathrm{S}^{\prime}, \mathrm{P}^{\prime}\right)$ if $\mathrm{S}^{\prime} \neq \varnothing, \quad ; \quad \mathrm{I}(\mathrm{P}, \mathrm{S}) \quad$ if $\mathrm{P} \neq \varnothing, \quad$ Bramantip

$\mathrm{A}\left(\mathrm{M}^{\prime}, \mathrm{P}^{\prime}\right) \mathrm{A}\left(\mathrm{M}^{\prime}, \mathrm{S}^{\prime}\right) \quad \mathrm{M}^{\prime}=\mathrm{S}^{\prime} \mathrm{P}^{\prime} \mathrm{M}^{\prime}$ no name. If $\mathrm{M}^{\prime} \neq \varnothing: \quad \mathrm{I}\left(\mathrm{S}^{\prime}, \mathrm{P}^{\prime}\right)$, No name $\mathrm{A}\left(\mathrm{M}^{\prime}, \mathrm{P}^{\prime}\right) \mathrm{I}\left(\mathrm{M}^{\prime}, \mathrm{S}^{\prime}\right) \quad \mathrm{S}^{\prime} \mathrm{P}^{\prime} \mathrm{M}^{\prime} \neq \varnothing$ or $\quad \mathrm{I}\left(\mathrm{S}^{\prime}, \mathrm{P}^{\prime}\right), \quad$ No name $\mathrm{I}\left(\mathrm{M}^{\prime}, \mathrm{P}^{\prime}\right) \mathrm{A}\left(\mathrm{M}^{\prime}, \mathrm{S}^{\prime}\right) \quad \mathrm{S}^{\prime} \mathrm{P}^{\prime} \mathrm{M}^{\prime} \neq \varnothing \quad$ or $\quad \mathrm{I}\left(\mathrm{S}^{\prime}, \mathrm{P}^{\prime}\right), \quad$ No name

Non-conclusive PCPs for $\mathrm{M}^{*}=\mathrm{M}^{\prime}, \mathrm{S}^{*}=\mathrm{S}^{\prime}, \mathrm{P}^{*}=\mathrm{P}^{\prime}$ :

$\mathrm{I}\left(\mathrm{M}^{\prime}, \mathrm{P}^{\prime}\right) \mathrm{I}\left(\mathrm{M}^{\prime}, \mathrm{S}^{\prime}\right), \quad \mathrm{I}\left(\mathrm{M}^{\prime}, \mathrm{P}^{\prime}\right) \mathrm{I}\left(\mathrm{M}, \mathrm{S}^{\prime}\right), \quad \mathrm{A}\left(\mathrm{P}^{\prime}, \mathrm{M}^{\prime}\right) \mathrm{I}\left(\mathrm{M}^{\prime}, \mathrm{S}^{\prime}\right), \quad \mathrm{I}\left(\mathrm{M}^{\prime}, \mathrm{P}^{\prime}\right) \mathrm{A}\left(\mathrm{S}^{\prime}, \mathrm{M}^{\prime}\right)$

Note the action of $\mathrm{G}$ on the set $\left\{\mathrm{H}_{1}, \mathrm{H}_{2}, \ldots, \mathrm{H}_{8}\right\}: \mathrm{s}\left(\mathrm{H}_{\mathrm{i}}\right)=\mathrm{H}_{\mathrm{i}+1}$, for $\mathrm{i}=1,3,5,7$, and, (since $\mathrm{s}^{2}=\mathrm{e}$ ), $\mathrm{s}\left(\mathrm{H}_{\mathrm{i}}\right)=\mathrm{H}_{\mathrm{i}-1}$, for $\mathrm{i}=2,4,6,8 ; \mathrm{p}\left(\mathrm{H}_{\mathrm{i}}\right)=\mathrm{H}_{\mathrm{i}+2}$, for $\mathrm{i}=1,2,5,6$, and, $\mathrm{p}\left(\mathrm{H}_{\mathrm{i}}\right)=\mathrm{H}_{\mathrm{i}-2}$, for 
$\mathrm{i}=3,4,7,8 ; \mathrm{m}\left(\mathrm{H}_{\mathrm{i}}\right)=\mathrm{H}_{\mathrm{i}+4}$, for $\mathrm{m}=1,2,3,4$, and, $\mathrm{m}\left(\mathrm{H}_{\mathrm{i}}\right)=\mathrm{H}_{\mathrm{i}-4}$, for $\mathrm{m}=5,6,7,8$. Also $\left\{\mathrm{G}\left(\mathrm{H}_{1}\right)\right\}=\ldots=\left\{\mathrm{G}\left(\mathrm{H}_{8}\right)\right\}=\left\{\mathrm{H}_{1}, \mathrm{H}_{2}, \mathrm{H}_{3}, \ldots, \mathrm{H}_{8}\right\}=\{$ All the 64 PCPs and their respective LCs if any $\}$. For example, $\left\{\mathrm{G}\left(\mathrm{H}_{1}\right)\right\}=\left\{\mathrm{e}\left(\mathrm{H}_{1}\right)=\mathrm{H}_{1}, \mathrm{~s}\left(\mathrm{H}_{1}\right)=\mathrm{H}_{2}, \mathrm{p}\left(\mathrm{H}_{1}\right)=\mathrm{H}_{3}, \mathrm{sp}\left(\mathrm{H}_{1}\right)=\mathrm{H}_{4}, \mathrm{~m}\left(\mathrm{H}_{1}\right)=\right.$ $\left.\mathrm{H}_{5}, \operatorname{sm}\left(\mathrm{H}_{1}\right)=\mathrm{H}_{6}, \mathrm{sp}\left(\mathrm{H}_{1}\right)=\mathrm{H}_{7}, \operatorname{spm}\left(\mathrm{H}_{1}\right)=\mathrm{H}_{8}\right\}=\{$ All the 64 PCPs and their respective LCs if any .)

The above shows that all the 64 PCPs are the result of replacing the positive $\mathrm{M}, \mathrm{P}$ and $\mathrm{S}$ terms from $\mathrm{H}_{1}$ with their negative counterparts in all the possible ways - thus obtaining the other $\mathrm{H}_{2}, \ldots, \mathrm{H}_{8}$ groups of eight PCPs each. One may say that, all the eight syllogisms of Barbara's type are in fact just one Barbara syllogism, whose content can be, and is, changed, via replacing some or all of its positive terms, S, P, M, with their negative counterparts. Similar assertions can be made about the other three groups of eight syllogisms each, of the Darapti, Darii and Disamis types: they all appear in the $\mathrm{H}_{1}$ and their content is varied by replacing their S,P,M positive terms, in all the possible ways, with their complementary sets in $U$ - the universe of discourse. In general, one may say that out of all the 32 conclusive syllogisms of types (1)-(3ii), one may choose any four - one syllogism per type - declare that those are the only syllogistic formats, and that all the variation of their logic content is accounted for, via changing their three terms between their positive and negative values. Thus, one may choose Barbara, Darapti, Darii and Disamis as syllogistic type representatives. Moreover, switching the roles played by the $\mathrm{S}$ and $\mathrm{P}$ terms in the Darii's type syllogisms one may obtain all the content of the Disamis type syllogisms from the Darii type syllogisms in which the roles of the $\mathrm{S}$ and $\mathrm{P}$ terms were interchanged: the $\mathrm{S}$, (resp. $\mathrm{P}$ ), term appears in the particular, (resp. universal), premise in the Darii type syllogisms, while the $S$, (resp. P), term appears in the universal, (resp. particular), premise, in the Disamis type syllogisms.)

One can also see that any conclusive syllogism of, say, Barbara's type, may be written as, e.g., a Barbara syllogism via term relabelings: in the formula (1), $\mathrm{A}\left(\mathrm{M}^{*}, \mathrm{P}^{*}\right) \mathrm{A}\left(\mathrm{S}^{*}, \mathrm{M}^{*}\right)$, replace $\mathrm{M}^{*}$ by $\mu, \mathrm{P}^{*}$ by $\pi$, and $\mathrm{S}^{*}$ by $\sigma$, and one obtains a Barbara syllogism in the variables $\mu, \pi, \sigma, \mathrm{A}(\mu, \pi) \mathrm{A}(\sigma, \mu)$, (equivalent to $\sigma \subseteq \mu \subseteq \pi$, and having the "All $\sigma$ is $\pi^{\prime}, \sigma=\sigma \mu \pi$ and $\pi^{\prime}=\pi^{\prime} \mu^{\prime} \sigma^{\prime}$ as LCs, and where, nevertheless, the content of the Barbara syllogism written in the variables $\mu, \pi, \sigma$ remains the same as the content before the relabeling: e.g., a Camestres syllogism, even if re-written as a Barbara syllogism, preserves its initial content). The variable domains are as follows: $\sigma$ and $S^{*} \in\left\{S, S^{\prime}\right\}, \pi$ and $P^{*} \in\left\{P, P^{\prime}\right\}, \mu$ and $M^{*} \in\left\{M, M^{\prime}\right\}$. Similarly, one could have written all the eight conclusive syllogisms of Barbara's type as either a Celarent syllogism (with the LCs, "No $\sigma$ is $\pi$ ", $\pi=\pi \mu^{\prime} \sigma^{\prime}, \sigma=\sigma \mu \pi^{\prime}$ ), or, as a Bramantip syllogism (with the universal LCs, “All $\pi$ is $\sigma^{\prime}, \pi=\pi \mu \sigma \sigma^{\prime}=\sigma^{\prime} \mu^{\prime} \pi^{\prime}$ ), etc. For example, Camestres, $\mathrm{A}(\mathrm{P}, \mathrm{M}) \mathrm{E}(\mathrm{S}, \mathrm{M})$, can be written, without any content change, as Bramantip via the relabeling $\mathrm{M}=\mu, \mathrm{P}=\pi, \mathrm{S}=\sigma^{\prime}: \mathrm{A}(\pi, \mu) \mathrm{A}(\mu, \sigma)$.

Vice versa, any particular conclusive syllogism of any of the four types (1)-(3ii), may be written as any other syllogism of the same type, via term relabelings. For example, Bramantip premises, $\mathrm{A}\left(\mathrm{M}^{\prime}, \mathrm{P}^{\prime}\right) \mathrm{A}\left(\mathrm{S}^{\prime}, \mathrm{M}^{\prime}\right)=\mathrm{A}(\mathrm{P}, \mathrm{M}) \mathrm{A}(\mathrm{M}, \mathrm{S})$, and their universal LCs, $\mathrm{P}=\mathrm{PMS}, \mathrm{S}^{\prime}=\mathrm{S}^{\prime} \mathrm{M}^{\prime} \mathrm{P}$ ', “All $\mathrm{P}$ is $\mathrm{S}$ ”, can be written, without a change in Bramantip's content, as any other syllogism of Barbara's type, $\mathrm{A}\left(\mu^{*}, \pi^{*}\right) \mathrm{E}\left(\sigma^{*}, \mu^{*}\right)$, via the relabeling $\mathrm{M}^{\prime}=\mu^{*}, \mathrm{P}^{\prime}=\pi^{*}, \mathrm{~S}^{\prime}=\sigma^{*}$. For example, Bramantip becomes Barbara in the $\mu, \pi, \sigma$ 
variables, $\mathrm{A}(\mu, \pi) \mathrm{A}(\sigma, \mu)$, via the relabeling $\mathrm{M}^{\prime}=\mu, \mathrm{P}^{\prime}=\pi, \mathrm{S}^{\prime}=\sigma$. The LCs of the latter Barbara, when translated back into the $\mathrm{S}, \mathrm{P}, \mathrm{M}$ variables, will still be the Bramantip LCs - whose content does not change when it is re-written as a Barbara syllogism.

The above observations may be formulated more intuitively as follows. Let's consider that a syllogism can change via changing its content, its format, or its terms, and these changes are interrelated. One may try to keep constant one of these three factors and change only the other two. From the Classical Syllogistic point of view the terms do not change - they are the fixed positive terms M, P, S. But the format, (and consequently the content), of the syllogisms can change according to the moods and figures of the syllogisms: Camestres is different from Barbara, and both differ from Disamis, etc. From a "pattern point of view" the format of a particular type of syllogisms does not change - for example, Barbara type syllogisms all satisfy the double inclusions' format $S^{*} \subseteq M^{*} \subseteq P^{*}$. In this case, what changes are the terms used in the pattern. The content of a particular type of syllogisms changes with the term triplets $\left(\mathrm{M}^{*}, \mathrm{P}^{*}, \mathrm{~S}^{*}\right)$ "plugged in" the pattern. One may start with the (M, P, S) triplet - "plugged in" any of the four syllogism types (or formats), and apply the eight transformations of the group G, (in its active interpretation), to obtain all the content changes of that syllogism format. Moreover, by switching the roles played by the $\mathrm{S}$ and $\mathrm{P}$ terms in the Darii type syllogisms, one may obtain (the content of) the Disamis type syllogisms. Finally, as shown above, and in accord with the passive interpretation of the G group as a group of relabelings, one may keep a constant content while writing a particular syllogism, (e.g., Darii), using relabeled terms, as any other syllogism of the types Darii or Disamis. (To re-write Darii as a Disamis, one has to relabel the P term in Darii as the $\mathrm{S}$ term to be used in Disamis, etc.)

Note also, that one may split in other ways the 64 PCP matrix into eight sets of eight PCPs each, according to the eight possible values which the triplet ( $\left.\mathrm{M}^{*}, \mathrm{P}^{*}, \mathrm{~S}^{*}\right)$ can have. For example, one may replace the A statements in the formulas (1)-(5ii), by E statements to obtain the equivalent formulas (1')-(5ii') below:

(1') (Type Barbara) $\mathrm{E}\left(\mathrm{M}^{*}, \mathrm{P}^{*}\right) \mathrm{E}\left(\mathrm{M}^{*}, \mathrm{~S}^{*}\right): \mathrm{S}^{*}=\mathrm{S}^{*} \mathrm{M}^{*} \mathrm{P}^{* \prime}, \mathrm{P}^{*}=\mathrm{P}^{*} \mathrm{M}^{*} \mathrm{~S}^{* \prime} ; \mathrm{A}\left(\mathrm{S}^{*}, \mathrm{P}^{* \prime}\right)$ $\left[=\mathrm{A}\left(\mathrm{P}^{*}, \mathrm{~S}^{*}\right)=\mathrm{E}\left(\mathrm{S}^{*}, \mathrm{P}^{*}\right)\right]$ - after $\mathrm{M}^{*}$ is dropped; $\mathrm{I}\left(\mathrm{S}^{*}, \mathrm{P}^{*}\right)$ - after ei on $\mathrm{S}^{*} ; \mathrm{I}\left(\mathrm{P}^{*}, \mathrm{~S}^{* \prime}\right)$ after ei on $\mathrm{P}^{*}$.

(2') (Type Darapti) E(M*,P*)E( $\left.M^{*}, \mathrm{~S}^{*}\right)$ : $\mathrm{M}^{*}=\mathrm{M}^{*} \mathrm{P} \mathrm{P}^{*} \mathrm{~S}^{* \prime}, \mathrm{A}\left(\mathrm{M}^{*}, \mathrm{P}^{*} \mathrm{~S}^{*}\right) ; \mathrm{M}^{*} \mathrm{P}{ }^{*} \mathrm{~S}^{* \prime}$ $\neq \varnothing$ if $\mathrm{M}^{*} \neq \varnothing$, or, $\mathrm{I}\left(\mathrm{S}^{*}, \mathrm{P}^{*}\right)$ if $\mathrm{M}^{*} \neq \varnothing$ and after $\mathrm{M}^{*}$ is eliminated.

There are eight distinct PCPs of type Darii, $\mathrm{E}\left(\mathrm{M}^{*}, \mathrm{P}^{*}\right) \mathrm{I}\left(\mathrm{M}^{*}, \mathrm{~S}^{*}\right)$, and eight distinct PCPs of type Disamis, $\mathrm{I}\left(\mathrm{M}^{*}, \mathrm{P}^{*}\right) \mathrm{E}\left(\mathrm{M}^{*}, \mathrm{~S}^{*}\right)$. The LCs are again obtained via the decompositions: $\varnothing \neq \mathrm{M} * \mathrm{~S} *=\mathrm{M} * \mathrm{~S} * \mathrm{P} *+\mathrm{M} * \mathrm{~S} * \mathrm{P} *{ }^{\prime}=\mathrm{M} * \mathrm{~S} * \mathrm{P} *{ }^{\prime}, \quad$ and, $\quad$ resp., $\varnothing \neq \mathrm{M} * \mathrm{P} *=$ $M * S * P *+M * S * ' P *=M * S * ' P *$ Thus:

(3i') (Type Darii) $\mathrm{E}\left(\mathrm{M}^{*}, \mathrm{P}^{*}\right) \mathrm{I}\left(\mathrm{M}^{*}, \mathrm{~S}^{*}\right): \mathrm{M}^{*} \mathrm{~S}^{*} \mathrm{P}^{*} \neq \varnothing ; \mathrm{I}\left(\mathrm{S}^{*}, \mathrm{P}^{*}\right)\left[=\mathrm{O}\left(\mathrm{S}^{*}, \mathrm{P}^{*}\right)\right]$ - after $\mathrm{M}^{*}$ is dropped from the $\mathrm{LC}$.

(3ii') (Type Disamis) I( $\left.\mathrm{M}^{*}, \mathrm{P}^{*}\right) \mathrm{E}\left(\mathrm{M}^{*}, \mathrm{~S}^{*}\right): \mathrm{M} * \mathrm{P}^{*} \mathrm{~S}^{*} \neq \varnothing ; \mathrm{I}\left(\mathrm{P}^{*}, \mathrm{~S}^{* \prime}\right)\left[=\mathrm{O}\left(\mathrm{P}^{*}, \mathrm{~S}^{*}\right)\right]$ - after $\mathrm{M}^{*}$ is dropped from the $\mathrm{LC}$.

(4i') $\mathrm{I}\left(\mathrm{M}^{*}, \mathrm{P}^{*}\right) \mathrm{I}\left(\mathrm{M}^{*}, \mathrm{~S}^{*}\right)$,

(4ii') $\mathrm{I}\left(\mathrm{M}^{*}, \mathrm{P}^{*}\right) \mathrm{I}\left(\mathrm{M}^{*}, \mathrm{~S}^{*}\right)$.

(5i') $\mathrm{E}\left(\mathrm{M}^{*}, \mathrm{P}^{*}\right) \mathrm{I}\left(\mathrm{M}^{*}, \mathrm{~S}^{*}\right)$, 
(5ii') $\mathrm{I}\left(\mathrm{M}^{*}, \mathrm{P}^{*}\right) \mathrm{E}\left(\mathrm{M}^{*}, \mathrm{~S}^{*}\right)$.

The set $\mathrm{K}_{1}$ of eight conclusive syllogisms and inconclusive PCPs obtained by choosing - in the formulas ( $\left.1^{\prime}\right)-\left(5 i^{\prime}\right)-M^{*}=M, P^{*}=P, S^{*}=S$, will now contain Celarent/Cesare and Ferio/Festino/Ferison/Fresison. The sets $\mathrm{K}_{2}, \mathrm{~K}_{3}, \ldots, \mathrm{K}_{8}$, may be obtained from the formulas $\left(1^{\prime}\right)-\left(5 i^{\prime}\right)$, by giving to the triplet $\left(\mathrm{M}^{*}, \mathrm{P}^{*}, \mathrm{~S}^{*}\right)$ the values (M, P, S'), (M, P', S), .., ( $\left.\mathrm{M}^{\prime}, \mathrm{P}^{\prime}, \mathrm{S}^{\prime}\right)$. The action of the group $\mathrm{G}$ on the set $\mathrm{K}_{1}, \mathrm{~K}_{2}, \ldots$, $\mathrm{K}_{8}$ mirrors the action of $\mathrm{G}$ on the sets $\mathrm{H}_{1}, \mathrm{H}_{2}, \ldots, \mathrm{H}_{8}: \mathrm{s}\left(\mathrm{K}_{\mathrm{i}}\right)=\mathrm{K}_{\mathrm{i}+1}$, for $\mathrm{i}=1,3,5,7$, and, (since $\mathrm{s}^{2}=\mathrm{e}$ ), $\mathrm{s}\left(\mathrm{K}_{\mathrm{i}}\right)=\mathrm{K}_{\mathrm{i}-1}$, for $\mathrm{i}=2,4,6,8 ; \mathrm{p}\left(\mathrm{K}_{\mathrm{i}}\right)=\mathrm{K}_{\mathrm{i}+2}$, for $\mathrm{i}=1,2,5,6$, and, $\mathrm{p}\left(\mathrm{K}_{\mathrm{i}}\right)=\mathrm{K}_{\mathrm{i}-2}$, for $\mathrm{i}=3,4,7,8 ; \mathrm{m}\left(\mathrm{K}_{\mathrm{i}}\right)=\mathrm{K}_{\mathrm{i}+4}$, for $\mathrm{m}=1,2,3,4$, and, $\mathrm{m}\left(\mathrm{K}_{\mathrm{i}}\right)=\mathrm{K}_{\mathrm{i}-4}$, for $\mathrm{m}=5,6,7,8$.

The next section adds more details about simple sorites of the Barbara, Darapti and Darii types.

\section{Biliteral Sorites}

The four types of PCPs which generate conclusive syllogisms may be immediately extended to simple sorites of the Barbara, Darapti, Darii types. Note that the latter type sorite has just one particular premise.

1. The premises of a simple Barbara type Aristotelian/Goclenian sorite are, SOR1:=E( $\left(\mathrm{S}^{*}, \mathrm{M}_{1}{ }^{*}\right) \mathrm{E}\left(\mathrm{M}_{1}{ }^{* \prime}, \mathrm{M}_{2}{ }^{*}\right) \mathrm{E}\left(\mathrm{M}_{2}{ }^{* \prime}, \mathrm{M}_{3}{ }^{*}\right) \ldots \mathrm{E}\left(\mathrm{M}_{\mathrm{i}}{ }^{\prime \prime}, \mathrm{M}_{1+1}{ }^{*}\right) \ldots \mathrm{E}\left(\mathrm{M}_{\mathrm{k}}{ }^{* \prime}, \mathrm{M}_{\mathrm{k}+1}{ }^{*}\right) \ldots$

$\mathrm{E}\left(\mathrm{M}_{\mathrm{n}}{ }^{\prime}, \mathrm{P} *\right)=\mathrm{A}\left(\mathrm{S}^{*}, \mathrm{M}_{1}{ }^{*}\right) \mathrm{A}\left(\mathrm{M}_{1}{ }^{* \prime}, \mathrm{M}_{2}{ }^{* \prime}\right) \mathrm{A}\left(\mathrm{M}_{2}{ }^{* \prime}, \mathrm{M}_{3}{ }^{* \prime}\right) \ldots \mathrm{A}\left(\mathrm{M}_{\mathrm{n}}{ }^{*}, \mathrm{P}^{* \prime}\right)$, with:

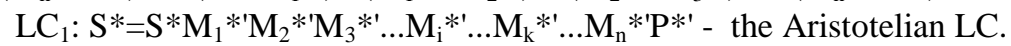

$\mathrm{LC}_{2}: \mathrm{P} * \mathrm{P} * \mathrm{M}_{\mathrm{n}} * \ldots \mathrm{M}_{\mathrm{k}} * \ldots \mathrm{M}_{\mathrm{i}} * \ldots \mathrm{M}_{3} * \mathrm{M}_{2} * \mathrm{M}_{1} * \mathrm{~S}^{*}=\mathrm{P} * \mathrm{M}_{1} * \mathrm{M}_{2} * \mathrm{M}_{3} * \ldots \mathrm{M}_{\mathrm{i}} * \ldots \mathrm{M}_{\mathrm{k}} * \ldots \mathrm{M}_{\mathrm{n}} * \mathrm{~S}^{*}$

- the Goclenian LC of the same premises. As in Barbara's PCP which contains just two chain links, $\mathrm{E}\left(\mathrm{S}, \mathrm{M}^{\prime}\right)$ and $\mathrm{E}(\mathrm{M}, \mathrm{P})$, the two LCs are not independent because they are based on two chains of inclusions which are deducible one from the other: $\mathrm{P}^{*} \subseteq \mathrm{M}_{\mathrm{n}}{ }^{*} \subseteq \ldots \subseteq \mathrm{M}_{3}{ }^{*} \subseteq \mathrm{M}_{2}{ }^{*} \subseteq \mathrm{M}_{1}{ }^{*} \subseteq \mathrm{S}^{* \prime}$ and $\left.\mathrm{S}{ }^{*} \subseteq \mathrm{M}_{1}{ }^{*} \subseteq \mathrm{M}_{2}{ }^{* \prime} \subseteq \mathrm{M}_{3}{ }^{*} \subseteq \ldots \subseteq \mathrm{M}_{\mathrm{n}}{ }^{\prime} \subseteq \mathrm{P}^{* \prime}.\right)$

2. The premises of a simple Darapti type sorite are,

SOR2:=E(S*, $\left.M^{*}\right) E\left(M_{1}{ }^{*}, M^{*}\right) E\left(M_{2}{ }^{*}, M^{*}\right) \ldots E\left(M_{i}{ }^{*}, M^{*}\right) E\left(M_{i+1} *, M^{*}\right) \ldots E\left(M_{n}{ }^{*}, M^{*}\right)$

$E\left(M^{*}, P *\right)$, with the $L C, M^{*}=M^{*} S^{*} M_{1}{ }^{*} M_{2}{ }^{*} M_{3}{ }^{*} \ldots M_{i}{ }^{\prime} M_{i+1}{ }^{*} \cdot \ldots M_{n}{ }^{*} P * '$, where, if $\mathrm{M}^{*} \neq \varnothing$, some of the many ei LCs are $\mathrm{MS}^{*} \mathrm{M}_{1}{ }^{\prime} \mathrm{M}_{2}{ }^{*} \mathrm{M}_{3}{ }^{*} \ldots \mathrm{M}_{\mathrm{i}}{ }^{*} \mathrm{M}_{\mathrm{i}+1}{ }^{*} \ldots \mathrm{M}_{\mathrm{n}}{ }^{* \prime} \mathrm{P}{ }^{* 1} \neq \varnothing$, $\mathrm{I}\left(\mathrm{S}^{*}, \mathrm{P}^{* \prime}\right)$, etc.

3. The premises of a simple Darii type sorite are:

SOR3:=E( $\left.S^{*}, M_{\mathrm{x}}{ }^{*}\right) \mathrm{E}\left(\mathrm{M}_{1}, \mathrm{M}_{\mathrm{y}}{ }^{*}\right) \mathrm{E}\left(\mathrm{M}_{2}{ }^{*}, \mathrm{M}_{\mathrm{y}}{ }^{*}\right) \ldots \mathrm{I}\left(\mathrm{M}_{\mathrm{x}}{ }^{*}, \mathrm{M}_{\mathrm{y}}{ }^{*}\right) \ldots \mathrm{E}\left(\mathrm{M}_{\mathrm{i}}{ }^{*}, \mathrm{M}_{\mathrm{y}}{ }^{*}\right) \mathrm{E}\left(\mathrm{M}_{\mathrm{i}+1}{ }^{*}, \mathrm{M}_{\mathrm{x}}{ }^{*}\right)$ $\ldots \mathrm{E}\left(\mathrm{M}_{\mathrm{n}} *, \mathrm{M}_{\mathrm{y}} *\right) \mathrm{E}\left(\mathrm{M}_{\mathrm{x}} *, \mathrm{P}^{*}\right)$

LC: $M_{x} * M_{y} * S * ' M_{1} * M_{2} *^{\prime} M_{3} * ' \ldots M_{i} * ' M_{i+1}{ }^{\prime} \ldots M_{n} *^{\prime} P * 1 \neq \varnothing$

Note that there are two types of "chains" making up the three sorites above. One is a D-chain, (or Darapti-chain), $\mathrm{E}(\alpha, \beta) \mathrm{E}(\alpha, \gamma) \mathrm{E}(\alpha, \delta)$, the other is a B-chain, (or Barbarachain), $\mathrm{E}(\mathrm{a}, \mathrm{b}) \mathrm{E}\left(\mathrm{b}^{\prime}, \mathrm{c}\right) \mathrm{E}\left(\mathrm{c}^{\prime}, \mathrm{d}\right)$, where $\alpha, \beta, \gamma, \delta, \mathrm{a}, \mathrm{b}, \mathrm{c}, \mathrm{d}$ are sets, $\alpha \cap \beta \cap \gamma \cap \delta$ is written $\alpha \beta \gamma \delta$, etc., and the LCs are $\alpha=\alpha \beta^{\prime} \gamma^{\prime} \delta^{\prime}$ and, respectively, $\mathrm{a}=\mathrm{ab} \mathrm{b}^{\prime} \mathrm{c}^{\prime} \mathrm{d}$ ' and $\mathrm{d}=\mathrm{dcba}$.

Note that in SOR3 one could have had just one D-chain instead of two, (for example, replace $\mathrm{M}_{\mathrm{y}}{ }^{*}$, everywhere but in $\mathrm{I}_{\mathrm{x}}\left(\mathrm{M}_{\mathrm{x}}{ }^{*}, \mathrm{M}_{\mathrm{y}}{ }^{*}\right)$, with $\mathrm{M}_{\mathrm{x}}{ }^{*}$ - to obtain just one Dchain), or one might have had one or two B-chains, (or one B-chain and one D-chain), originating at $\mathrm{M}_{\mathrm{x}}{ }^{*}$ or/and $\mathrm{M}_{\mathrm{y}}{ }^{*}$. 
To the two 3-links B and D-chains just above, one may add many other premises/chains and still have the extended premises entailing at least one LC:

$\mathrm{E}(\mathrm{a}, \mathrm{b}) \mathrm{E}\left(\mathrm{b}^{\prime}, \mathrm{c}\right) \mathrm{E}\left(\mathrm{c}^{\prime}, \mathrm{d}\right) \mathrm{E}\left(\mathrm{a}, \mathrm{b}_{1}\right) \mathrm{E}\left(\mathrm{b}_{1}{ }^{\prime}, \mathrm{c}_{1}\right) \mathrm{E}\left(\mathrm{c}_{1}{ }^{\prime}, \mathrm{d}_{1}\right) \ldots \mathrm{E}\left(\mathrm{a}, \mathrm{b}_{\mathrm{n}}\right) \mathrm{E}\left(\mathrm{b}_{\mathrm{n}}{ }^{\prime}, \mathrm{c}_{\mathrm{n}}\right) \mathrm{E}\left(\mathrm{c}_{\mathrm{n}}{ }^{\prime}, \mathrm{d}_{\mathrm{n}}\right)$, entails the LC $\mathrm{a}=a b^{\prime} c^{\prime} d^{\prime} b_{1}{ }^{\prime} c_{1}{ }^{\prime} d_{1}{ }^{\prime} \ldots b_{n}{ }^{\prime} c_{n}{ }^{\prime} d_{n}{ }^{\prime}$, after all $a=a b_{k}{ }^{\prime} c_{k}{ }^{\prime} d_{k}{ }^{\prime}, k=1,2, \ldots, n$, are substituted in (the initial) $\mathrm{a}=\mathrm{ab} \mathrm{c}^{\prime} \mathrm{d}^{\prime}$

If in the above premises one replaces all $d_{i} i=1,2, \ldots, n$, by $d$, then the $L C$ becomes $a=a b^{\prime} c^{\prime} d b_{1}{ }^{\prime} c_{1}{ }^{\prime} \ldots b_{n}{ }^{\prime} c_{n}{ }^{\prime}$, and there is a second LC: $d=d a a^{\prime} b c b_{1} c_{1} d_{1} \ldots b_{n} c_{n}$.

Another "extended premises" possibility, would have been to start the B-chains not at the term a, but at any other term, e.g., $c^{\prime}$ :

$\mathrm{E}(\mathrm{a}, \mathrm{b}) \mathrm{E}\left(\mathrm{b}^{\prime}, \mathrm{c}\right) \mathrm{E}\left(\mathrm{c}^{\prime}, \mathrm{d}\right) \mathrm{E}\left(\mathrm{c}^{\prime}, \mathrm{b}_{1}\right) \mathrm{E}\left(\mathrm{b}_{1}{ }^{\prime}, \mathrm{c}_{1}\right) \mathrm{E}\left(\mathrm{c}_{1}{ }^{\prime}, \mathrm{d}_{1}\right) \mathrm{E}\left(\mathrm{c}^{\prime}, \mathrm{b}_{2}\right) \mathrm{E}\left(\mathrm{b}_{2}{ }^{\prime}, \mathrm{c}_{2}\right) \mathrm{E}\left(\mathrm{c}_{2}{ }^{\prime}, \mathrm{d}_{2}\right) \ldots \mathrm{E}\left(\mathrm{c}^{\prime}, \mathrm{b}_{\mathrm{n}}\right) \mathrm{E}\left(\mathrm{b}_{\mathrm{n}}{ }^{\prime}\right.$, $\left.c_{n}\right) E\left(c_{n}{ }^{\prime}, d_{n}\right)$, entails the LC $a=a b^{\prime} c^{\prime} d^{\prime} b_{1}{ }^{\prime} c_{1}{ }^{\prime} d_{1}{ }^{\prime} \ldots b_{n}{ }^{\prime} c_{n}{ }^{\prime} d_{n}{ }^{\prime}$, obtained by substituting the $c^{\prime}$ term in $a=a b^{\prime} c^{\prime} d$ with $c^{\prime}=c^{\prime} b_{1}{ }^{\prime} c_{1}{ }^{\prime} d_{1}{ }^{\prime} \ldots b_{n}{ }^{\prime} c_{n}{ }^{\prime} d_{n}{ }^{\prime}$. If one would have started some $B$-chains at $\mathrm{c}^{\prime}$, and some other B-chains, (or a D-chain), at c, then no LC pointing to the unique subset of the entire universe of discourse, U, would have been possible. A "substitution principle" is necessary to hold if it were that a set of premises entails an LC: the partial LCs of all the partial B and D-chains have to allow term substitutions until all the partial LCs "unite" into an LC pinpointing just one subset of the entire universe of discourse U, comprising all the terms appearing in all the premises. All the subsets of $\mathrm{U}$ can be represented on a rectangular Karnaugh map drawn for subsets, not truth values (for which a toroidal $\mathrm{K}$-map is preferable).

Other examples (involving more indexes):

One may say that each of the three (or four) type sorites admits "Darapti or/and Barbara decorations", i.e., a D-chain or/and B-chain sequence of premises may be added using any term/letter as the "starting base" - exactly as the term (or letter) M was used as the "base" of SOR2 - the Darapti type (2) sorite above.

For example, if to SOR1 one adds Darapti decorations of length=1 on $\mathrm{S}^{*}, \mathrm{M}_{1}{ }^{*}$, and $\mathrm{P}^{* \prime}$, respectively, and a Darapti decoration of length $=3$ on $\mathrm{M}_{2}{ }^{*}$, i.e., if one adds these premises: $E\left(S^{*}, M_{n+1}{ }^{*}\right) E\left(M_{1}^{* \prime}, M_{n+2}{ }^{*}\right) E\left(M_{2}{ }^{\prime}, M_{n+3}{ }^{*}\right) E\left(M_{2}{ }^{\prime}, M_{n+4}{ }^{*}\right) E\left(M_{2}{ }^{\prime}\right.$, $\left.M_{n+5}{ }^{*}\right) E\left(P^{* \prime}, M_{n+6}^{*}\right)$, then one obtains a sorite which has just one LC not two. This $\mathrm{LC}$ is an "extension" of $\mathrm{LC}_{1}: \mathrm{S}^{*}=\mathrm{S}^{*} \mathrm{M}_{1}{ }^{*} \mathrm{M}_{2}{ }^{*} \mathrm{M}_{3}{ }^{*} \ldots \quad \mathrm{M}_{\mathrm{n}}{ }^{*} \mathrm{P}{ }^{*} \mathrm{M}_{\mathrm{n}+1}{ }^{*} \mathrm{M}_{\mathrm{n}+2}{ }^{* \prime}$ $M_{n+3}{ }^{\prime} M_{n+4}{ }^{\prime} M_{n+5} *^{\prime} M_{n+6} *^{\prime}$.

For the Goclenian reading of SOR1 one needs to add, as Darapti decorations of the same lengths as above, e.g., these premises:

$E\left(S^{* \prime}, M_{n+1}^{*}\right) E\left(M_{1}^{*}, M_{n+2} *\right) E\left(M_{2}^{*}, M_{n+3} *\right) E\left(M_{2}^{*}, M_{n+4} *\right) E\left(M_{2}^{*}, M_{n+5} *\right) E\left(P^{*}\right.$, $\mathrm{M}_{\mathrm{n}+6} *$, and then, again, one obtains a sorite which has just one LC not two. This LC

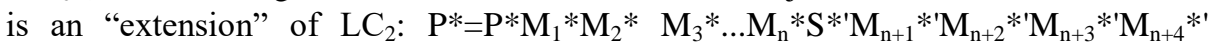
$\mathrm{M}_{\mathrm{n}+5}{ }^{\prime} \mathrm{M}_{\mathrm{n}+6}{ }^{*}$.

One may add Darapti decorations to a Darapti type sorite, SOR2, via adding, e.g., these premises:

$E\left(S^{* \prime}, M_{n+1}^{*}\right) E\left(M_{1}^{* \prime}, M_{n+2}{ }^{*}\right) E\left(M_{2}^{* \prime}, M_{n+3}{ }^{*}\right) E\left(M_{2}^{* \prime}, M_{n+4}{ }^{*}\right) E\left(M_{2}{ }^{\prime}, M_{n+5}{ }^{*}\right) E\left(P^{* \prime}\right.$, $\mathrm{M}_{\left.\mathrm{n}+6^{*}\right)}$.

Then one obtains a sorite whose LC is an "extension" of the above SOR2 LC:

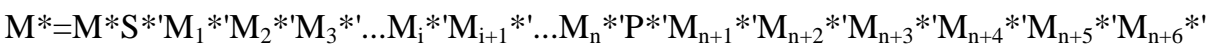


Analogously, Darapti decorations of the same lengths as above may be added to a Darii type sorite, SOR3, via adding these premises:

$E\left(S^{* \prime}, M_{n+1}{ }^{*}\right) E\left(M_{1}^{* \prime}, M_{n+2}{ }^{*}\right) E\left(M_{2}^{* \prime}, M_{n+3}{ }^{*}\right) E\left(M_{2}^{* \prime}, M_{n+4}{ }^{*}\right) E\left(M_{2}{ }^{\prime}, M_{n+5}{ }^{*}\right) E\left(P^{* \prime}\right.$, $\left.\mathrm{M}_{\mathrm{n}+6} *\right)$.

Then one obtains a sorite whose LC is an "extension" of the SOR3 LC: $\mathrm{M}_{\mathrm{x}} * \mathrm{M}_{\mathrm{y}}$

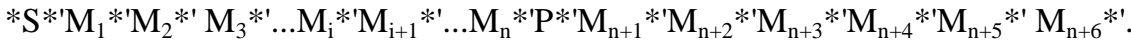

One thus sees that to any term/letter appearing in the LC of a sorite of types 1,2 or 3, one may add a "Darapti decoration", i.e., a (no matter how long) Darapti type sequence of premises with that letter as the "base", exactly as the term (or letter) M was the "base" of the Darapti type SOR2. "Barbara decorations" can be added in a similar way.

If represented on a subsets' Karnaugh map with enough variables, the LCs of the above SOR1, (resp. SOR2), say that $\mathrm{S}^{*}, \mathrm{P}^{*}$, (resp. $\mathrm{M}^{*}$ ), were reduced by the universal premises to just one, possibly not empty, partitioning subset of the universal set U. The LC of SOR3 affirms that there exists a partition subset of the universal set $U$ which is not empty.

A product shorthand notation of SOR1 would have as factors the chain links $E\left(M_{i}^{* \prime}, M_{i+1}{ }^{*}\right)$, where $i$ runs from 0 to $n$, and where $M_{0} *^{\prime \prime}=S, M_{n+1}{ }^{*}=P$. In this notation one can see that a B-chain can split, at any link i, into any number of new chains, originating either at $\mathrm{M}_{\mathrm{i}}{ }^{* \prime}$ or at $\mathrm{M}_{\mathrm{i}+1}$. For example, one may add to the SOR1 above, either premises which "come back in a loop" to the initial B-chain, such as in (a), $\mathrm{E}\left(\mathrm{M}_{\mathrm{i}}{ }^{* \prime}, \alpha_{1}\right) \mathrm{E}\left(\alpha_{1}{ }^{\prime}, \alpha_{2}\right) \mathrm{E}\left(\alpha_{2}{ }^{\prime}, \alpha_{3}\right) \mathrm{E}\left(\alpha_{3}{ }^{\prime}, \mathrm{M}_{\mathrm{k}}{ }^{*}\right)$, or continue with other partitioning sets $\alpha_{4}, \alpha_{5}$, etc., from the universal set $\mathrm{U}$, as in (b), $\mathrm{E}\left(\mathrm{M}_{\mathrm{i}}{ }^{* \prime}, \alpha_{1}\right) \mathrm{E}\left(\alpha_{1}^{\prime}, \alpha_{2}\right) \mathrm{E}\left(\alpha_{2}{ }^{\prime}, \alpha_{3}\right) \mathrm{E}\left(\alpha_{3}{ }^{\prime}, \alpha_{4}\right)$ $\mathrm{E}\left(\alpha_{4}^{\prime}, \alpha_{5}\right) \mathrm{E}\left(\alpha_{5}^{\prime}, \alpha_{6}\right)$. Or, new $B$-chains can originate at $\mathrm{M}_{\mathrm{i}+1} *$ : (c) $\mathrm{E}\left(\mathrm{M}_{\mathrm{i}+1}{ }^{*}, \beta_{1}\right) \mathrm{E}\left(\beta_{1}{ }^{\prime}, \beta_{2}\right) \mathrm{E}\left(\beta_{2}{ }^{\prime}, \beta_{3}\right) \mathrm{E}\left(\beta_{3}{ }^{\prime}, \mathrm{M}_{\mathrm{k}}{ }^{\prime \prime}\right)$ - these are premises which "come back in a

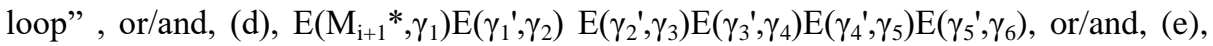
$\mathrm{E}\left(\mathrm{M}_{\mathrm{i}+1}{ }^{*}, \delta_{1}\right) \mathrm{E}\left(\delta_{1}{ }^{\prime}, \delta_{2}\right) \mathrm{E}\left(\delta_{2}{ }^{\prime}, \delta_{3}\right) \mathrm{E}\left(\delta_{3}{ }^{\prime}, \delta_{4}\right) \mathrm{E}\left(\delta_{4}^{\prime}, \delta_{5}\right)$. As before, the "extended" SOR1a, SOR1b, SOR1c, SOR1d, and SOR1e, entail an LC only if, (as a result of all the new and old (universal) premises being applied), one of the $n$ terms has $2^{n-1}-1$ empty subsets and only one possibly non-empty subset of the new, $2^{\mathrm{n}}$ "extended" partitioning of $\mathrm{U}$, determined by all the terms, old and new, contained in all the premises, old and new - or "extended".

For example, the extended sorite,

SOR $1 \mathrm{a}=\mathrm{E}\left(\mathrm{S}^{*}, \quad \mathrm{M}_{1}{ }^{*}\right) \mathrm{E}\left(\mathrm{M}_{1}{ }^{\prime \prime}, \quad \mathrm{M}_{2}{ }^{*}\right) \mathrm{E}\left(\mathrm{M}_{2}{ }^{* \prime}, \quad \mathrm{M}_{3}{ }^{*}\right) \ldots \mathrm{E}\left(\mathrm{M}_{\mathrm{i}}{ }^{\prime}, \quad \mathrm{M}_{\mathrm{i}+1}{ }^{*}\right) \ldots \mathrm{E}\left(\mathrm{M}_{\mathrm{k}}{ }^{\prime \prime}\right.$, $\left.\mathrm{M}_{\mathrm{k}+1}{ }^{*}\right) \ldots \mathrm{E}\left(\mathrm{M}_{\mathrm{n}}{ }^{*}, \mathrm{P}^{*}\right) \quad \mathrm{E}\left(\mathrm{M}_{\mathrm{i}}{ }^{* 1}, \alpha_{1}\right) \mathrm{E}\left(\alpha_{1}{ }^{\prime}, \alpha_{2}\right) \mathrm{E}\left(\alpha_{2}{ }^{\prime}, \alpha_{3}\right) \mathrm{E}\left(\alpha_{3}{ }^{\prime}, \mathrm{M}_{\mathrm{k}}{ }^{*}\right)$, still entails two LCs, $\mathrm{LC}_{1 \mathrm{a}}: \mathrm{S} *=\mathrm{S}^{*} \mathrm{M}_{1}{ }^{*} \mathrm{M}_{2}{ }^{*} \mathrm{M}_{3}{ }^{*}{ }^{\prime} \ldots \mathrm{M}_{\mathrm{i}}{ }^{*}{ }^{\prime} \ldots \mathrm{M}_{\mathrm{k}}{ }^{*} \ldots \mathrm{M}_{\mathrm{n}}{ }^{*} \mathrm{P}{ }^{* 1} \alpha_{1}{ }^{\prime} \alpha_{2}{ }^{\prime} \alpha_{3}{ }^{\prime}$ (the Aristotelian LC obtained after $M_{i}{ }^{*}=M_{i}{ }^{* \prime} \alpha_{1}{ }^{\prime} \alpha_{2}{ }^{\prime} \alpha_{3}{ }^{\prime} M_{k}{ }^{* \prime}$ was substituted in the $\mathrm{LC}_{1}$ of SOR1).

$\mathrm{LC}_{2 \mathrm{a}}: \mathrm{P} *=\mathrm{P} * \mathrm{M}_{\mathrm{n}} * \ldots \mathrm{M}_{\mathrm{k}} * \ldots \mathrm{M}_{\mathrm{i}} * \ldots \mathrm{M}_{3} * \mathrm{M}_{2} * \mathrm{M}_{1} * \mathrm{~S} * \alpha_{1} \alpha_{2} \alpha_{3}$ (the Goclenian LC obtained after $\mathrm{M}_{\mathrm{k}} *=\mathrm{M}_{\mathrm{k}} * \alpha_{1} \alpha_{2} \alpha_{3} \mathrm{M}_{\mathrm{i}} *$ was substituted in the $\mathrm{LC}_{2}$ of SOR1). The same is true for SOR1c - it will still admit two LCs: one has to substitute $\mathrm{M}_{\mathrm{i}+1}$ * in $\mathrm{LC}_{2}$, and $\mathrm{M}_{\mathrm{k}}{ }^{* \prime}$ in $\mathrm{LC}_{1}$ - see below. But note that if the last link of the "extension" (a) would have been $\mathrm{E}\left(\alpha_{3}{ }^{\prime}, \mathrm{M}_{\mathrm{k}}{ }^{* \prime}\right)$ instead of $\mathrm{E}\left(\alpha_{3}{ }^{\prime}, \mathrm{M}_{\mathrm{k}}{ }^{*}\right)$, then the substitution $\mathrm{M}_{\mathrm{i}}{ }^{* \prime}=\mathrm{M}_{\mathrm{i}}{ }^{\prime \prime} \alpha_{1}{ }^{\prime} \alpha_{2}{ }^{\prime} \alpha_{3}{ }^{\prime} \mathrm{M}_{\mathrm{k}}{ }^{*}$ in $\mathrm{LC}_{1 \mathrm{a}}$ would have produced the empty set: $\mathrm{S} * \mathrm{~S} * \mathrm{M}_{1}{ }^{\prime} \mathrm{M}_{2}{ }^{*} \mathrm{M}_{3}{ }^{*} \ldots \mathrm{M}_{\mathrm{i}}{ }^{\prime} \ldots \mathrm{M}_{\mathrm{k}}{ }^{*} \mathrm{M}_{\mathrm{k}}{ }^{*}{ }^{\prime} \ldots \mathrm{M}_{\mathrm{n}}{ }^{\prime} \mathrm{P}^{*}{ }^{*} \alpha_{1}{ }^{\prime} \alpha_{2}{ }^{\prime} \alpha_{3}{ }^{\prime}$. And no substitution would have been available to the Goclenian solution, $\mathrm{LC}_{2}$ of SOR1, to account for the exten- 
sion $\mathrm{M}_{\mathrm{k}}{ }^{\prime \prime}=\mathrm{M}_{\mathrm{k}}{ }^{*} \alpha_{1} \alpha_{2} \alpha_{3} \mathrm{M}_{\mathrm{i}}{ }^{*}$, while substituting $\mathrm{M}_{\mathrm{k}}{ }^{* \prime}$ into $\mathrm{LC}_{1}$ would have resulted again in an empty set.

The extended sorite,

SOR $1 b=E\left(S^{*}, \quad M_{1}^{*}\right) E\left(M_{1}^{* \prime}, \quad M_{2}^{*}\right) E\left(M_{2}{ }^{\prime \prime}, \quad M_{3}^{*}\right) \ldots E\left(M_{i}^{* \prime}, \quad M_{i+1}{ }^{*}\right) \ldots E\left(M_{k}{ }^{\prime}\right.$, $\left.\mathrm{M}_{\mathrm{k}+1}{ }^{*}\right) \ldots \mathrm{E}\left(\mathrm{M}_{\mathrm{n}}{ }^{* \prime}, \mathrm{P}^{*}\right) \quad \mathrm{E}\left(\mathrm{M}_{\mathrm{i}}{ }^{* \prime}, \alpha_{1}\right) \mathrm{E}\left(\alpha_{1}^{\prime}, \alpha_{2}\right) \mathrm{E}\left(\alpha_{2}{ }^{\prime}, \alpha_{3}\right) \mathrm{E}\left(\alpha_{3}{ }^{\prime}, \alpha_{4}\right) \mathrm{E}\left(\alpha_{4}^{\prime}, \alpha_{5}\right) \mathrm{E}\left(\alpha_{5}{ }^{\prime}, \alpha_{6}\right)$, entails only one $\mathrm{LC}, \mathrm{LC}_{\mathrm{bb}}$ : $\mathrm{S} * \mathrm{~S} * \mathrm{M}_{1}{ }^{\prime} \mathrm{M}_{2}{ }^{*} \mathrm{M}_{3}{ }^{*} \ldots \mathrm{M}_{\mathrm{i}}{ }^{\prime} \ldots \mathrm{M}_{\mathrm{k}}{ }^{\prime} \ldots \mathrm{M}_{\mathrm{n}}{ }^{*} \mathrm{P}{ }^{* \prime} \alpha_{1}{ }^{\prime} \alpha_{2}{ }^{\prime} \alpha_{3}{ }^{\prime} \alpha_{4}{ }^{\prime} \alpha_{5}{ }^{\prime} \alpha_{6}{ }^{\prime} \quad$ (the Aristotelian LC), while the Goclenian LC of SOL1, LC $\mathrm{P}^{*}=\mathrm{P} * \mathrm{M}_{\mathrm{n}} * \ldots \mathrm{M}_{\mathrm{k}} * \ldots \mathrm{M}_{\mathrm{i}} * \ldots \mathrm{M}_{3} * \mathrm{M}_{2} * \mathrm{M}_{1} * \mathrm{~S}^{* \prime}$, together with $\alpha_{6}=\alpha_{6} \alpha_{5} \alpha_{4} \alpha_{3} \alpha_{2} \alpha_{1} \mathrm{M}_{\mathrm{i}} *$ do not pinpoint a unique subset of U. A Goclenian $\mathrm{LC}_{2 \mathrm{~b}}$ does not exist.

As already mentioned, the extended sorite SOR1c, similar to SOR1a, entails two LCs,

SOR1c $=E\left(S^{*}, \quad M_{1}{ }^{*}\right) E\left(M_{1}^{* \prime}, \quad M_{2}^{*}\right) E\left(M_{2}^{* \prime}, \quad M_{3} *\right) \ldots E\left(M_{i}^{* \prime}, \quad M_{i+1}{ }^{*}\right) \ldots E\left(M_{k}{ }^{* \prime}\right.$, $\left.\mathrm{M}_{\mathrm{k}+1}{ }^{*}\right) \ldots \mathrm{E}\left(\mathrm{M}_{\mathrm{n}}{ }^{\prime}, \mathrm{P}^{*}\right) \quad \mathrm{E}\left(\mathrm{M}_{\mathrm{i}+1}{ }^{*}, \beta_{1}\right) \mathrm{E}\left(\beta_{1}{ }^{\prime}, \beta_{2}\right) \mathrm{E}\left(\beta_{2}{ }^{\prime}, \beta_{3}\right) \mathrm{E}\left(\beta_{3}{ }^{\prime}, \mathrm{M}_{\mathrm{k}}{ }^{* \prime}\right)$,

$\mathrm{LC}_{1 \mathrm{c}}: \mathrm{S} *=\mathrm{S}^{*} \mathrm{M}_{1}{ }^{*} \mathrm{M}_{2}{ }^{*} \mathrm{M}_{3} *{ }^{\prime} \ldots \mathrm{M}_{\mathrm{i}}{ }^{*} \ldots \mathrm{M}_{\mathrm{k}}{ }^{*} \ldots \mathrm{M}_{\mathrm{n}}{ }^{*} \mathrm{P}{ }^{* \prime} \beta_{1} \beta_{2} \beta_{3} \quad$ (the Aristotelian

LC, obtained after substituting $\mathrm{M}_{\mathrm{k}}{ }^{\prime}=\mathrm{M}_{\mathrm{k}}{ }^{\prime} \beta_{1} \beta_{2} \beta_{3} \mathrm{M}_{\mathrm{i}+1}{ }^{\prime \prime}$ in $\left.\mathrm{LC}_{1}\right)$.

$\mathrm{LC}_{2 \mathrm{c}}: \mathrm{P} *=\mathrm{P} * \mathrm{M}_{\mathrm{n}} * \ldots \mathrm{M}_{\mathrm{k}} * \ldots \mathrm{M}_{\mathrm{i}} * \ldots \mathrm{M}_{3} * \mathrm{M}_{2} * \mathrm{M}_{1} * \mathrm{~S}^{*} \beta_{1}{ }^{\prime} \beta_{2}{ }^{\prime} \beta_{3}{ }^{\prime}$ (the Goclenian LC, obtained after substituting $\mathrm{M}_{\mathrm{i}+1} *=\mathrm{M}_{\mathrm{i}+1} * \beta_{1}{ }^{\prime} \beta_{2}{ }^{\prime} \beta_{3}{ }^{\prime} \mathrm{M}_{\mathrm{k}} *$ in $\mathrm{LC}_{1}$ ).

The extended sorite,

SOR1bd=E( $\left.S^{*}, \quad M_{1}^{*}\right) E\left(M_{1}^{* \prime}, \quad M_{2}^{*}\right) E\left(M_{2}^{* \prime}, \quad M_{3}^{*}\right) \ldots E\left(M_{i}^{* \prime}, \quad M_{i+1}{ }^{*}\right) \ldots E\left(M_{k} *^{\prime}\right.$, $\left.\mathrm{M}_{\mathrm{k}+1}{ }^{*}\right) \ldots \mathrm{E}\left(\mathrm{M}_{\mathrm{n}}{ }^{* \prime}, \quad \mathrm{P}^{*}\right) \quad \mathrm{E}\left(\mathrm{M}_{\mathrm{i}}{ }^{* \prime}, \alpha_{1}\right) \mathrm{E}\left(\alpha_{1}^{\prime}, \alpha_{2}\right) \mathrm{E}\left(\alpha_{2}^{\prime}, \alpha_{3}\right) \mathrm{E}\left(\alpha_{3}^{\prime}, \alpha_{4}\right) \quad \mathrm{E}\left(\alpha_{4}^{\prime}, \alpha_{5}\right) \mathrm{E}\left(\alpha_{5}^{\prime}, \alpha_{6}\right)$ $\mathrm{E}\left(\mathrm{M}_{\mathrm{i}+1}{ }^{*}, \gamma_{1}\right) \mathrm{E}\left(\gamma_{1}^{\prime}, \gamma_{2}\right) \mathrm{E}\left(\gamma_{2}{ }^{\prime}, \gamma_{3}\right) \mathrm{E}\left(\gamma_{3}^{\prime}, \gamma_{4}\right) \mathrm{E}\left(\gamma_{4}^{\prime}, \gamma_{5}\right)$, does not have any $\mathrm{LC}$, since the two "LC partial extensions" $M_{i}{ }^{*}=M_{i}{ }^{*} \alpha_{1}{ }^{\prime} \alpha_{2}{ }^{\prime} \alpha_{3}{ }^{\prime} \alpha_{4}{ }^{\prime} \alpha_{5}{ }^{\prime} \alpha_{6}{ }^{\prime}$ and $M_{i+1} *=M_{i+1} * \gamma_{1}{ }^{\prime} \gamma_{2} \gamma_{3} \gamma_{4} \gamma_{5}{ }^{\prime}$ can not be simultaneously substituted in either $\mathrm{LC}_{1}$ or $\mathrm{LC}_{2}$ of SOR1. Finally, the extended sorite,

SOR1de $=E\left(S^{*}, \quad M_{1}^{*}\right) E\left(M_{1}^{* \prime}, \quad M_{2}^{*}\right) E\left(M_{2}^{* \prime}, \quad M_{3}^{*}\right) \ldots E\left(M_{i}^{* \prime}, \quad M_{i+1}{ }^{*}\right) \ldots E\left(M_{k}^{* \prime}\right.$, $\left.\mathrm{M}_{\mathrm{k}+1}{ }^{*}\right) \ldots \mathrm{E}\left(\mathrm{M}_{\mathrm{n}}{ }^{*}, \mathrm{P}^{*}\right) \mathrm{E}\left(\mathrm{M}_{\mathrm{i}+1}{ }^{*}, \gamma_{1}\right) \mathrm{E}\left(\gamma_{1}^{\prime}, \gamma_{2}\right) \mathrm{E}\left(\gamma_{2}^{\prime}, \gamma_{3}\right) \mathrm{E}\left(\gamma_{3^{\prime},}, \gamma_{4}\right) \mathrm{E}\left(\gamma_{4^{\prime}}, \gamma_{5}\right) \mathrm{E}\left(\mathrm{M}_{\mathrm{i}+1}{ }^{*}, \delta_{1}\right) \mathrm{E}\left(\delta_{1}^{\prime}, \delta_{2}\right)$ $\mathrm{E}\left(\delta_{2}{ }^{\prime}, \delta_{3}\right) \mathrm{E}\left(\delta_{3}{ }^{\prime}, \delta_{4}\right) \mathrm{E}\left(\delta_{4}{ }^{\prime}, \delta_{5}\right)$, has one LC only - $\mathrm{LC}_{1 \mathrm{de}}$ does not exist since neither of the "extended partial" LCs, $M_{i+1} *=M_{i+1}{ }^{*} \gamma_{1}{ }^{\prime} \gamma_{2}{ }^{\prime} \gamma_{3} \gamma_{4}{ }^{\prime} \gamma_{5}{ }^{\prime}$ and $M_{i+1} *=M_{i+1} * \delta_{1}{ }^{\prime} \delta_{2}{ }^{\prime} \delta_{3}{ }^{\prime} \delta_{4}{ }^{\prime} \delta_{5}$ ' can be substituted in the $\mathrm{LC}_{1}, \mathrm{~S} *=\mathrm{S}^{*} \mathrm{M}_{1}{ }^{\prime} \mathrm{M}_{2}{ }^{*} \mathrm{M}_{3}{ }^{*} \ldots \mathrm{M}_{\mathrm{i}}{ }^{*} \ldots \mathrm{M}_{\mathrm{k}}{ }^{*} \ldots \mathrm{M}_{\mathrm{n}}{ }^{* \prime} \mathrm{P}{ }^{* \prime}$, of SOR1.

But, via substitutions, one obtains

$\mathrm{LC}_{2 \mathrm{de}}: \quad \mathrm{P} *=\mathrm{P} * \mathrm{M}_{\mathrm{n}} * \ldots \mathrm{M}_{\mathrm{k}} * \ldots \mathrm{M}^{*} * \ldots \mathrm{M}_{3} * \mathrm{M}_{2} * \mathrm{M}_{1} * \mathrm{~S}^{*} \gamma^{\prime} \gamma_{1} \gamma_{2}{ }^{\prime} \gamma_{3}{ }^{\prime} \gamma_{4} \gamma_{5}{ }^{\prime} \delta_{1}{ }^{\prime} \quad \delta_{2}{ }^{\prime} \delta_{3}{ }^{\prime} \delta_{4}{ }^{\prime} \delta_{5}{ }^{\prime} \quad$, the Goclenian LC of SOR1).

Anywhere in the D-chain of SOR2 one may have added an arbitrary number of Bchains, such as the above: (a) $\mathrm{E}\left(\mathrm{M}_{\mathrm{i}}{ }^{* \prime}, \alpha_{1}\right) \mathrm{E}\left(\alpha_{1}{ }^{\prime}, \alpha_{2}\right) \mathrm{E}\left(\alpha_{2}{ }^{\prime}, \alpha_{3}\right) \mathrm{E}\left(\alpha_{3}{ }^{\prime}, \mathrm{M}_{\mathrm{k}}{ }^{*}\right)$, or/and (b), $\mathrm{E}\left(\mathrm{M}_{\mathrm{i}}{ }^{* \prime}, \alpha_{1}\right) \mathrm{E}\left(\alpha_{1}^{\prime}, \alpha_{2}\right) \mathrm{E}\left(\alpha_{2}^{\prime}, \alpha_{3}\right) \mathrm{E}\left(\alpha_{3}^{\prime}, \alpha_{4}\right) \quad \mathrm{E}\left(\alpha_{4}^{\prime}, \alpha_{5}\right) \mathrm{E}\left(\alpha_{5}^{\prime}, \alpha_{6}\right), \quad$ or/and $\quad$ (c') $\mathrm{E}\left(\mathrm{M}_{\mathrm{i}+1}{ }^{* \prime}, \beta_{1}\right) \mathrm{E}\left(\beta_{1}^{\prime}, \beta_{2}\right) \mathrm{E}\left(\beta_{2}^{\prime}, \beta_{3}\right) \quad \mathrm{E}\left(\beta_{3}^{\prime}, \mathrm{M}_{\mathrm{k}}{ }^{*}\right), \quad$ or/and, $\left(\mathrm{d}^{\prime}\right), \quad \mathrm{E}\left(\mathrm{M}_{\mathrm{i}+1}{ }^{* \prime}, \gamma_{1}\right)$ $\mathrm{E}\left(\gamma_{1}^{\prime}, \gamma_{2}\right) \mathrm{E}\left(\gamma_{2}^{\prime}, \gamma_{3}\right) \mathrm{E}\left(\gamma_{3^{\prime}}, \gamma_{4}\right) \quad \mathrm{E}\left(\gamma_{4}^{\prime}, \gamma_{5}\right), \quad$ or/and, $\quad\left(\mathrm{e}^{\prime}\right), \quad \mathrm{E}\left(\mathrm{M}_{\mathrm{i}+1}{ }^{* \prime}, \delta_{1}\right) \mathrm{E}\left(\delta_{1}^{\prime}, \delta_{2}\right)$ $\mathrm{E}\left(\delta_{2}{ }^{\prime}, \delta_{3}\right) \mathrm{E}\left(\delta_{3}{ }^{\prime}, \delta_{4}\right) \mathrm{E}\left(\delta_{4}{ }^{\prime}, \delta_{5}\right)$, since all the "extended partial" LCs, $\mathrm{M}_{\mathrm{i}}{ }^{* \prime}=\mathrm{M}_{\mathrm{i}}{ }^{* \prime} \alpha_{1}{ }^{\prime} \alpha_{2}{ }^{\prime} \alpha_{3}{ }^{\prime} \mathrm{M}_{\mathrm{k}}{ }^{* \prime}, \quad \mathrm{M}_{\mathrm{i}}{ }^{\prime}=\mathrm{M}_{\mathrm{i}}{ }^{*} \alpha_{1}{ }^{\prime} \alpha_{2}{ }^{\prime} \alpha_{3}{ }^{\prime} \alpha_{4}{ }^{\prime} \alpha_{5}{ }^{\prime} \alpha_{6}{ }^{\prime}, \quad \mathrm{M}_{\mathrm{i}+1}{ }^{*}=\mathrm{M}_{\mathrm{i}+1}{ }^{*} \gamma_{1} \gamma_{2}{ }^{\prime} \quad \gamma_{3}{ }^{\prime} \gamma_{4}{ }^{\prime} \gamma_{5}{ }^{\prime}$, and $\mathrm{M}_{\mathrm{i}+1}{ }^{*}=\mathrm{M}_{\mathrm{i}+1}{ }^{*} \delta_{1}{ }^{\prime} \delta_{2}{ }^{\prime} \delta_{3}{ }^{\prime} \delta_{4}{ }^{\prime} \delta_{5}{ }^{\prime}$ can be substituted in the LC of SOR2. 


\section{Conclusions}

The extension of classical syllogistic to the 32 conclusive syllogisms with indefinite terms and the interpretation of the terms as sets, evidentiate the three patterns followed by all the conclusive syllogisms and the fact that their logical consequences (LCs) point a unique partitioning subset of the universe of discourse, $\mathrm{U}$.

One saw that, based on set inclusions and set intersections, one may say that only three distinct patterns (or formats) of conclusive syllogisms do exist - they represent the $n=3$ case of the $n$ terms biliteral sorites of the Barbara, Darapti and Darii patterns. The premises of the latter pattern imply that a unique partitioning subset of the universe of discourse, $\mathrm{U}$, is known to be non-empty. The premises of the Barbara and Darapti patterns imply that the "smallest" set (or term), i.e., the term included in all the other terms (or sets) is empty, except for a unique partitioning subset of the universe of discourse, U, which is part of that "smallest" set, and which might remain, possibly, non-empty. The "pattern and type" classification of conclusive syllogisms can be used to simplify the Rules of Valid Syllogisms. This "pattern and type classification" also shows that all the content variation of the conclusive syllogisms of the same type is obtained by substituting, (into any of the particular term permutations of a given pattern), all the $2^{3}$ indefinite $\left(M^{*}, P^{*}, S^{*}\right)$ term triplets - where $S^{*} \in\left\{S, S^{\prime}\right\}$, $\mathrm{P}^{*} \in\left\{\mathrm{P}, \mathrm{P}^{\prime}\right\}, \mathrm{M}^{*} \in\left\{\mathrm{M}, \mathrm{M}^{\prime}\right\}$. A "pattern and type" presentation of Classical Syllogistics can replace its traditional presentation - where the term values are kept constant, (i.e. equal to their positive values $\mathrm{M}, \mathrm{P}, \mathrm{S}$ ), and the content of the syllogisms varies in accord with the moods and figures of the valid syllogisms.

\section{References}

1. Reichenbach, Hans. The syllogism revised. Philosophy of Science, 19(1):1-16, January 1952.

2. Churchman, C. W.: Elements of Logic and Formal Science. J. B. Lippincott, Chicago, 1940.

3. Smith, H. B.: A Primer of Logic. B. D. Smith \& bros., Pulaski, Va., 1917.

4. Pratt, Vaughan: The four essential Aristotelian syllogisms, via substitution and symmetry. https://stanford.academia.edu/VaughanPratt/Drafts.

5. Alvarez, Enrique, Correia, Manuel: Syllogistic with Indefinite Terms. History and Philosophy of Logic 33 (4):297-306. (2012). DOI: 10.1080/01445340.2012.680704

6. Jevons, W. Stanley: The Substitution Of Similars, The True Principle Of Reasoning, Derived From A Modification Of Aristotle 's Dictum. Macmillan And Co., London (1869). Reprint Edition: Wentworth Press (2019). 